\title{
Non-fibro-adipogenic pericytes from human embryonic stem cells attenuate degeneration of the chronically injured mouse muscle
}

\author{
Gina M. Mosich, ${ }^{1}$ Regina Husman, ${ }^{1}$ Paras Shah, ${ }^{1}$ Abhinav Sharma, ${ }^{1}$ Kevin Rezzadeh, ${ }^{1}$ \\ Temidayo Aderibigbe, ${ }^{1}$ Vivian J. Hu, ${ }^{1}$ Daniel J. McClintick, ${ }^{1}$ Genbin Wu, ${ }^{1}$ Jonathan D. Gatto, ${ }^{1}$ \\ Haibin Xi, ${ }^{2}$ April D. Pyle, ${ }^{2}$ Bruno Péault, ${ }^{1,2,3}$ Frank A. Petrigliano, ${ }^{4}$ and Ayelet Dar ${ }^{1}$ \\ 'Orthopaedic Hospital Research Center, David Geffen School of Medicine, and ${ }^{2} E l i$ and Edythe Broad Center for \\ Regenerative Medicine and Stem Cell Research, UCLA, California, USA. ${ }^{3}$ Center for Cardiovascular Science and MRC \\ Centre for Regenerative Medicine, University of Edinburgh, Edinburgh, United Kingdom. ${ }^{4}$ Epstein Family Center for Sports \\ Medicine, Keck School of Medicine of the University of Southern California, Los Angeles, California, USA.
}

\begin{abstract}
Massive tears of the rotator cuff (RC) are associated with chronic muscle degeneration due to fibrosis, fatty infiltration, and muscle atrophy. The microenvironment of diseased muscle often impairs efficient engraftment and regenerative activity of transplanted myogenic precursors. Accumulating myofibroblasts and fat cells disrupt the muscle stem cell niche and myogenic cell signaling and deposit excess disorganized connective tissue. Therefore, restoration of the damaged stromal niche with non-fibro-adipogenic cells is a prerequisite to successful repair of an injured RC. We generated from human embryonic stem cells (hES) a potentially novel subset of PDCFR$\beta^{+} C D 146+C D 34-C D 56-$ pericytes that lack expression of the fibro-adipogenic cell marker PDCFR- $\alpha$. Accordingly, the PDGFR- $\beta^{+}$PDGFR- $\alpha^{-}$phenotype typified non-fibro-adipogenic, non-myogenic, pericyte-like derivatives that maintained non-fibro-adipogenic properties when transplanted into chronically injured murine RCs. Although administered hES pericytes inhibited developing fibrosis at early and late stages of progressive muscle degeneration, transplanted PDGFR- $\boldsymbol{\beta}^{+}$PDGFR- $\boldsymbol{\alpha}^{+}$ human muscle-derived fibro-adipogenic progenitors contributed to adipogenesis and greater fibrosis. Additionally, transplanted hES pericytes substantially attenuated muscle atrophy at all tested injection time points after injury. Coinciding with this observation, conditioned medium from cultured hES pericytes rescued atrophic myotubes in vitro. These findings imply that non-fibroadipogenic $\mathrm{hES}$ pericytes recapitulate the myogenic stromal niche and may be used to improve cell-based treatments for chronic muscle disorders.
\end{abstract}

Authorship note: $\mathrm{BP}, \mathrm{FAP}$, and $\mathrm{AD}$ are co-senior authors.

Conflict of interest: A patent application (30435.354-US-P2) has been filed by $B P, F A P$, and $A D$ on a method for generation of therapeutic pericytes from human pluripotent stem cells.

Copyright: () 2019, American Society for Clinical Investigation.

Submitted: October 4, 2018

Accepted: October 31, 2019

Published: December 19, 2019

Reference information: /CI Insight.

2019;4(24):e125334.

https://doi.org/10.1172/jci.

insight.125334.

\section{Introduction}

The rotator cuff $(\mathrm{RC})$ is a group of muscles and associated tendons that are essential for shoulder stability and motion. RC tears are an exceedingly common musculoskeletal condition, the prevalence of which is estimated to be up to $50 \%$ of the US population and higher in older individuals (1). To date, RC injury research has largely focused on improving healing at the bone-tendon interface in an attempt to repair the native enthesis, while the treatment of muscle atrophy and fibro-adipogenic degeneration observed in the setting of large RC tears has been largely ignored. This is despite the fact that both atrophy and fibro-adipogenic degeneration of the muscle have been correlated with poor functional outcome and decreased strength following RC repair (2) and shown to be irreversible even in the setting of complete tendon-to-bone healing (3). Muscle atrophy and fibro-adipogenic degeneration may impair tendon mechanics and diminish compliance of the myotendinous unit, which ultimately diminishes functionality of the repaired RC (4-6). This characteristic fibro-adipogenic degeneration seen following massive RC tears is unique to RC muscle and not commonly observed in other skeletal muscles. Increased lipid deposition and fibrosis diminish tendon compliance, and these changes appear irreversible following repair (2). Furthermore, atrophy of the RC musculature is associated with loss of strength and contractile amplitude, resulting in shoulder weakness and 
poor function (7). Although surgical repair for small tears may yield acceptable clinical outcomes, repair of large tears is complicated by an unacceptably high re-tear rate. Myogenic cells have demonstrated promise in the regeneration of chronically injured RC (8). However, fibrosis in diseased organs often disrupts efficient engraftment of functional progenitors because of a hostile microenvironment. For instance, the myogenicity of satellite cells may be compromised in the fibrotic muscle (9). Therefore, treatments to reverse muscle fibro-adipogenesis seem to be a prerequisite for transplantation of myo-regenerative cells. Muscular fibroadipogenic degeneration originates in PDGFR- $\alpha^{+}$precursor cells present in the perivascular spaces of injured muscles (10). PDGFR- $\beta$, which is expressed by pericytes, has also been identified on activated fibro-adipogenic precursors in the lung, kidney, and liver (11). We have recently demonstrated that fibro-adipogenic precursors are typified by coexpression of PDGFR- $\beta$ and PDGFR- $\alpha$ in cardiac and skeletal muscles $(12,13)$. These results suggest a close developmental and anatomic relationship between native perivascular myo-regenerative cells and fibro-adipogenic progenitors. Consequently, transplantation of heterogenous mesodermal precursors, such as culture-expanded mesenchymal stem cells (14), which are also of perivascular origin (15), creates the risk of replenishing the degenerating muscle with PDGFR- $\alpha^{+}$fibro-adipogenic cells that may promote fibrotic and adipogenic tissue degeneration. Therefore, improved stem cell-based therapies, such as replenishment of the diseased stromal niche with regenerative cells to repair microenvironment damage, may facilitate engraftment of myogenic precursors and induce long-lasting myo-regeneration that is necessary for successful muscle healing. Furthermore, these transplanted cells should not contribute to fibro-adipogenesis that may impair myogenic regeneration.

Human pluripotent (embryonic or induced) stem cells (hPSCs) provide a plentiful source for generation of diverse cell types. Multiple protocols to differentiate hPSCs into mesenchymal stem-like cells (16) and perivascular cells (17-19) have been described. However, functional differentiation demonstrated that in most cases, hPSC mesodermal derivatives are heterogenous cell populations composed of a mixture of mesodermal progenitors that can be distinguished based on their differentiation potentials (e.g., osteo-/chondro-/ fibro-/adipogenic) and level of expression or lack of expression of characteristic sets of markers $(20,21)$. Additionally, in vitro modifications evolve in long-term cultures, resulting in progressive cell maturation or acquirement of fibroblast-like characteristics (12). Accordingly, we have developed a protocol for generation of PDGFR- $\beta^{+}$PDGFR- $\alpha^{-}$pericytes from human embryonic stem cells ( $\mathrm{hES}$ ), characterized their differentiation properties, and tested their therapeutic potential in the degenerated muscle using a clinically relevant mouse model of chronic RC tears $(8,13,22)$. We were able to generate large numbers of pericyte-like cells with a simple and reproducible protocol. A potentially novel marker combination, PDGFR- $\beta^{+}$PDGFR- $\alpha^{-}$, defines non-fibro-adipogenic lineage-restricted pericytes (LR-PCs) that coexpress canonical pericyte markers. When transplanted into the chronically injured mouse RC, engrafted LR-PCs maintained their intrinsic non-fibro-adipogenic features in the degenerating environment and indirectly supported myo-regeneration. When administered at 2 and 6 weeks after injury, the time points that are correlated with development of fibrosis in injured RCs, LR-PCs effectively attenuated excess production of collagens. On the contrary, administered human muscle-derived fibro-adipogenic progenitors (FAPs) increased fibrosis in injured muscles regardless of the timing of injection. Additionally, myofiber atrophy, which is observed within a few days after injury and progresses for weeks, was markedly reduced at all tested time points of LR-PC administration, as compared with the lack of effect on muscle atrophy when FAPs were injected.

\section{Results}

Chronic injury of the murine RC muscle leads to fibro-adipogenic degeneration. Transection of the murine suprascapular nerve and supraspinatus tendons (TTDN) results in chronic injury of the RC that mirrors the clinical outcome in humans, characterized by progressive muscle atrophy and massive fibro-adipogenic degeneration. As we have previously demonstrated $(8,13)$, examination of RC histological sections revealed stage-specific degenerative remodeling of the injured muscle in comparison with non-injured (Figure 1A) and sham-operated RC (Figure 1B) at different time points after injury (Figure 1, A-F). Massive necrosis of myofibers and a rapid increase in muscle cellularity, indicative of acute inflammation and cell proliferation, was observed within 4 days after TTDN (Figure 1C). Transient muscle regeneration at 2 weeks after TTDN (Figure 1D) was accompanied by the development of fibro-adipocytic tissue (Figure 1, D and J) and progressed to massive muscle degeneration due to replacement of atrophied muscle fibers by adipose tissue (Figure 1E) and development of collagen-producing fibrous scars (Figure 1, G-K) within 6 weeks after TTDN. The severity of muscle deterioration further increased at 10 weeks after TTDN with larger 

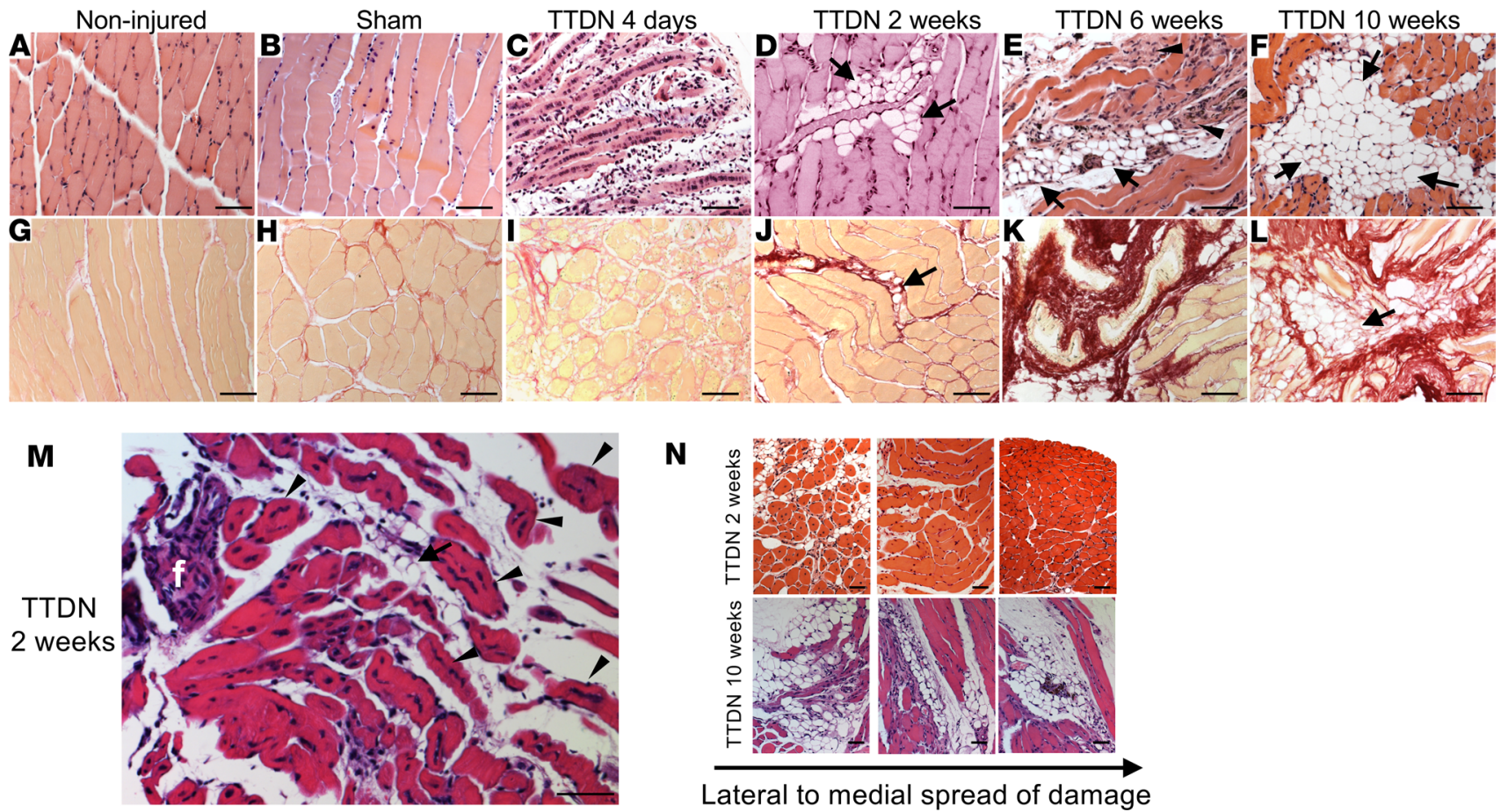

Figure 1. Stage-specific degeneration of chronically injured RC muscle. (A-F) Representative H\&E images of changes in normal skeletal muscle of RC (A) at the indicated time points after transection of the tendons and nerve (TTDN) and compared with sham-operated RC (B). (C) A short transition phase of significant increase in muscle cellularity was seen at 4 days after TTDN. Within 2 weeks transient muscle regeneration was accompanied by developing fatty infiltration of adipocytes (D, arrows). At 6 weeks (E) and 10 weeks (F) after TTDN, chronic muscle damage was characterized by severe muscle atrophy and increasing amounts of adipose tissue (arrows) and fibrosis (arrowheads). (G-L) Representative images of picrosirius red staining of collagen in sections of non-operated (G) and sham-operated RC (H) or 4 days (I), 2 weeks (J), 6 weeks (K), and 10 weeks (L) after TTDN. (M) Muscle regeneration at 2 weeks after TTDN is indicated by the presence of central multinuclei, non-atrophied myofibers (arrowheads) surrounding developing fat tissue (arrow) and fibrotic scar (f). (N) Lateral to medial imaging (from left to right) of H\&E-stained RC sections at 2 weeks or 10 weeks after TTDN. Scale bars: $100 \mu$ m.

adipocyte clusters (Figure 1D) and fibrotic areas (Figure 1L). Changes in muscle structure and myofiber regeneration were observed at the lateral side at 2 weeks after TTDN (Figure 1, M and N), and muscle damage increased and spread in a medial direction in the muscle belly over time (Figure $1 \mathrm{~N}$ ). Increasing fibro-adipogenic stimulus may alter the activity of transplanted therapeutic cells from regeneration to degeneration. In an attempt to overcome this problem, we aimed to generate stromal cell-like progenitors that are resistant to pro-fibro-adipogenic cues.

Derivation of non-fibro-adipogenic CD $146^{+} P D G F R-\alpha^{-}$cells from $h E S$. Progenitor cells were generated by prolonged induction of hES differentiation in endothelial growth medium. Flow cytometry analysis revealed robust generation of $\mathrm{CD} 146^{+} \mathrm{CD} 45^{-} \mathrm{CD} 34^{-}$cells with an efficiency of up to $90 \%$ cell differentiation within 14 days (Figure 2A). hES derivatives were then sorted by multicolor flow cytometry based on the expression of CD146 (perivascular, endothelial, and mesenchymal cell marker) and lack of expression of CD34 (hematopoietic, endothelial, and adventitial cell marker) and CD45 (hematopoietic cell marker) and were rapidly expanded in endothelial cell growth medium (EGM-2). Alternatively, expanded CD146 ${ }^{+} \mathrm{CD} 45$ $\mathrm{CD}^{-} 4^{-}$cells dominated the culture at passage 2 without the need for cell sorting. Similar to pericytes (PCs) from spontaneously differentiating hPSCs (17), and cultured FACS-sorted human native PCs (15), hES derivatives stably expressed perivascular cell markers when expanded in culture (from passage 2 and up to passage 9), including CD146, PDGFR- $\beta$, and CD73, but did not express CD34 and CD45 (Figure 2A). Regardless of cell passage, hES CD $146^{+} \mathrm{CD} 34^{-} \mathrm{CD} 45^{-} \mathrm{PCs}$ exhibited vasculogenic features when seeded in Matrigel, rearranging into tubular networks (Figure 2B). Mesenchymal stem cells have been the most commonly used therapeutic cells for immunomodulation and reconstitution of diseased tissues $(23,24)$ and were also tested for their ability to promote regeneration in chronically injured RC muscle (25). However, the heterogeneity of conventional culture-derived mesenchymal stem cells increases the risk of replenishing the degenerating muscle with PDGFR- $\beta^{+}$PDGFR- $\alpha^{+}$fibro-adipogenic mesodermal precursors $(10,12,13)$ that will promote tissue degeneration. Flow cytometry analysis of coexpression of PDGFR- $\alpha$ with CD34, 
A
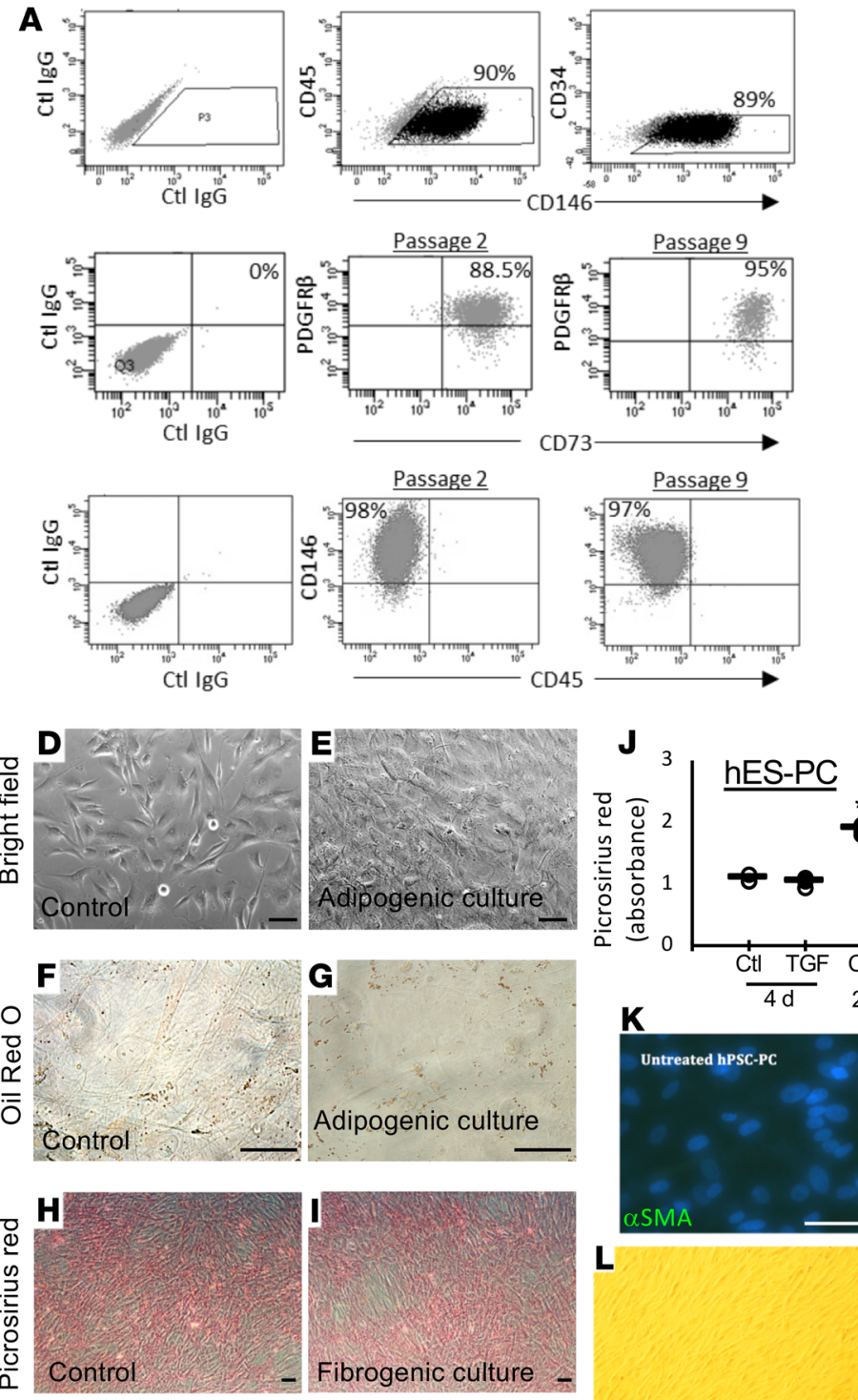

$\mathbf{L}$
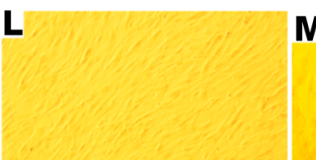

Control
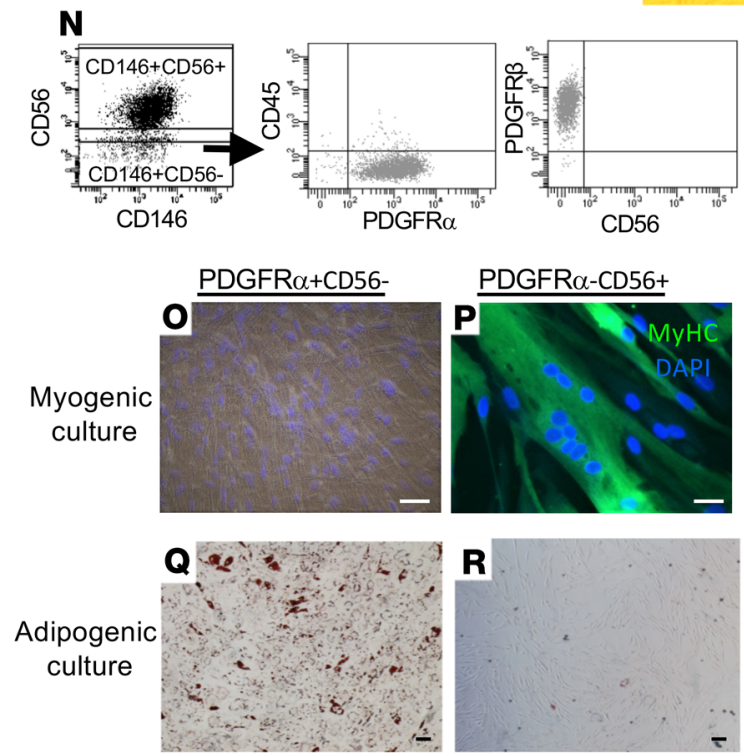
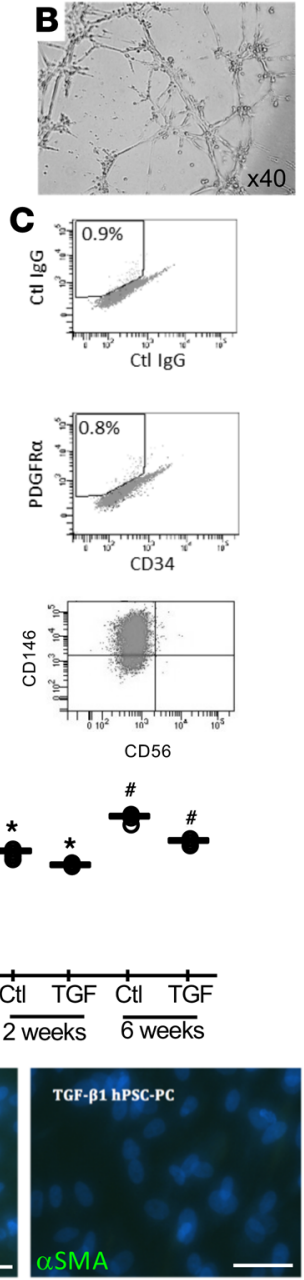

M

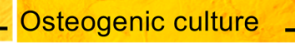

Control

S

\section{byso} $(\mathbf{O}-\mathbf{R})$ Myogenic ( $\mathbf{O}$ and $\mathbf{P})$ and adipogenic $(\mathbf{Q}$ and $\mathbf{R}$ ) cultures of PDCFR- $\alpha^{+} C^{-} 556^{-}(\mathbf{O}$ and $\mathbf{Q})$ and PDCFR- $\alpha^{-}{ }^{-C D 56} 6^{+}$(P and $\mathbf{R}$ ) cells. (S) Concentration of collagen in control and TGF- $\beta 1-$ induced PDGFR- $\alpha^{+}$CD56- cell cultures (mean \pm SEM). Data were pooled from 3 independent experiments ( $n=3$ donors) with triplicates. ${ }^{*} P<0.005$ compared with untreated cultures (1-way ANOVA). Scale bars: $100 \mu \mathrm{m}$.

Figure 2. Human embryonic stem cell derivatives are lineage-restricted pericytes. (A) Representative flow cytometry dot plot of CD34, CD45, and CD146 expression by hES cell derivatives expanded in EGM-2 medium at passage 0 . Flow cytometry analysis of expression of characteristic cell surface markers: PDCFR- $\beta$, CD73, and CD146 (pericytic); CD45 (hematopoietic); and CD34 (endothelial, hematopoietic progenitors and adventitial cells) by expanded hES derivafe at passages 2 and 9. (B) Vasculogenic demonstrated by tube formation on Matrigel. (c) Representative dot plots of flow cytometry analysis of CD34, PDCFR- $\alpha$, CD146, and expression by expanded hES pericytic derivatives. (D-K) hES PCs lack fibro-adipoare not detected in control (D) and adipogenic cultures (E). Negative Oil Red 0 staining of non-induced control (F) and adipogenic induced cultures (G). Representative images of picrosirius red staining for collagen of control (H) and TCF- $\beta 1$-treated (I) hES PC cultures. Spectrophotometric quantification of collagen production (mean \pm SEM) by non-induced and TCF- $\beta 1$-induced hES PC cultures at the indicated time points $(\mathrm{J}) .{ }^{\#} P<0.00001$ compared with control and TCF- $\beta 1$ induced hES PCs that were cultured for 4 days and 2 and TCF- $\beta 1$-induced hES PCs that were cultured for 4 days (1-way ANOVA). TCF- $\beta 1$ does not induce the expression of $\alpha$-smooth muscle actin (K, $\alpha$-SMA in green, nuclear staining for DAPI in blue) by hES PCs. (L and $\mathbf{M}$ ) Poor staining for alizarin red demonstrates limited osteogenic differentiation of induced hES PCs. (N) Sorting strategy based on the expression of CD146 and CD56 by human muscle cells expanded in EGM-2 medium at passage 0 . Flow cytometry analysis of the expression of PDCFR- $\beta$, PDCFR- $\alpha$, and CD45

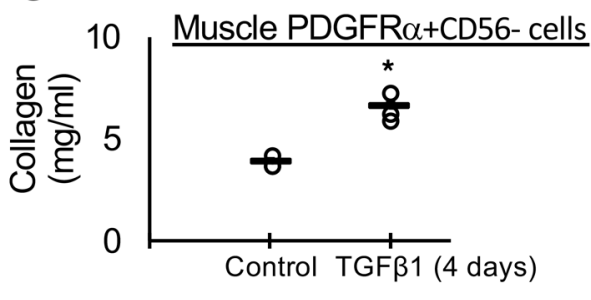


A

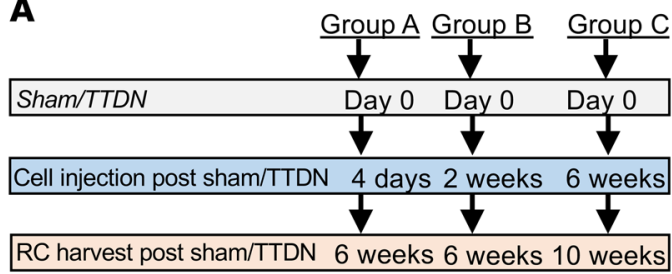

B

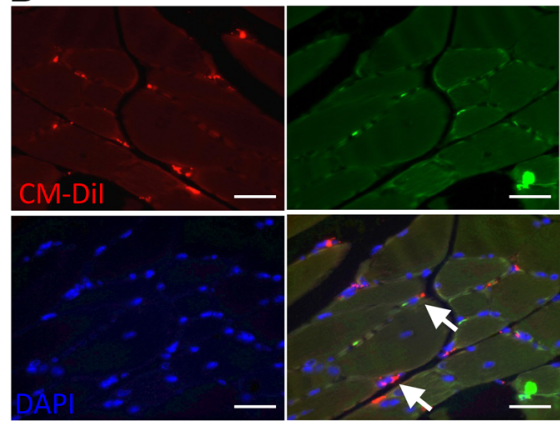

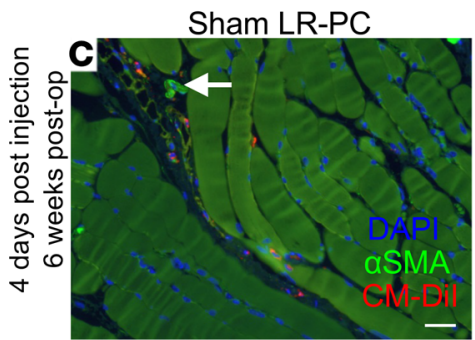

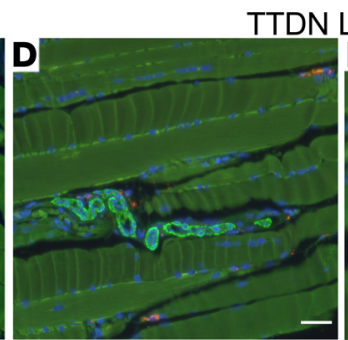

TTDN LR-PC
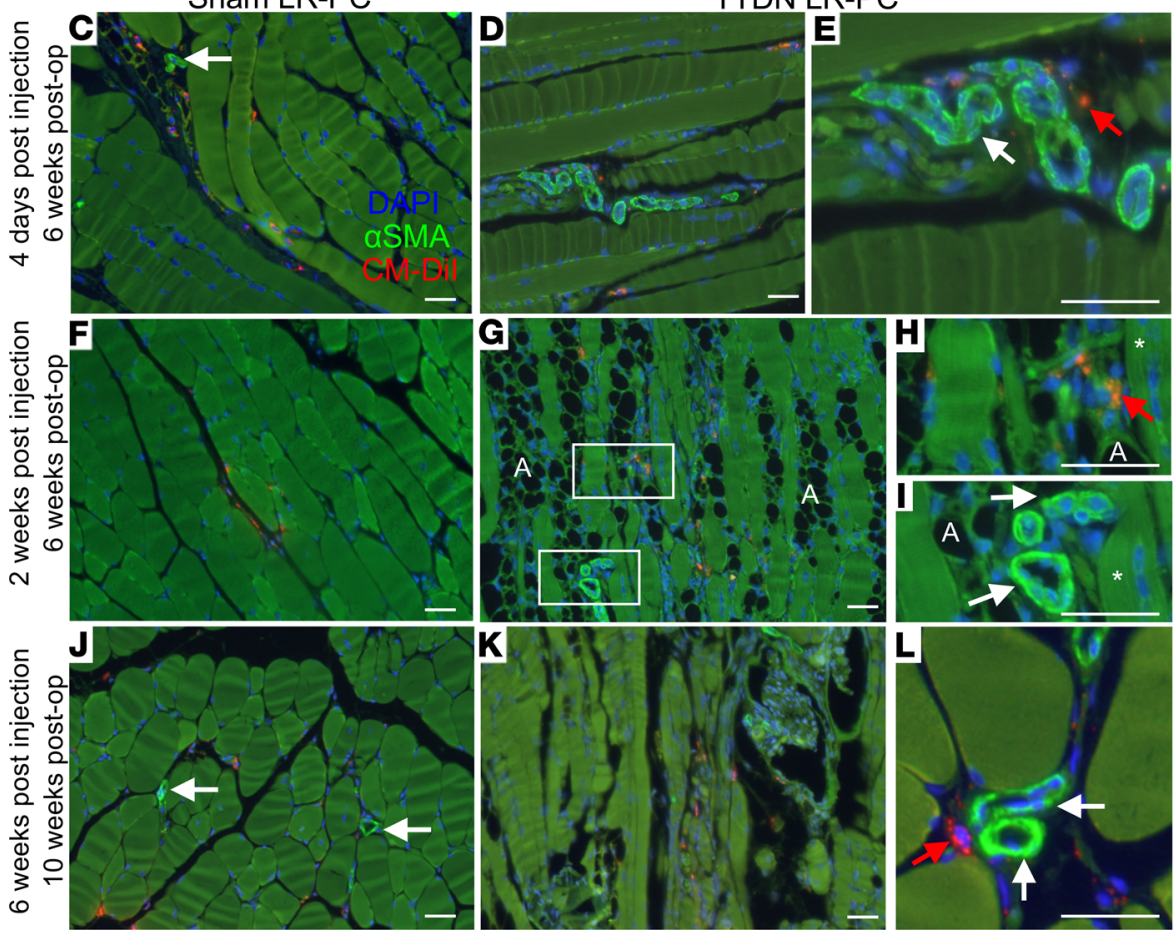

Figure 3. Distribution of transplanted LR-PCs in chronically injured RC. (A) Illustration of experimental design of LR-PC or control human muscle FAP transplantation at progressive stages of RC fibro-adipogenesis. (B-L) Fluorescence and $\alpha$-SMA immunofluorescence representative images of CM-Dil+ (red) LR-PC distribution in sham-operated or injured RC administered at 4 days, 2 weeks, and 6 weeks after surgery (groups A-C). CM-Dil+ cells are seen aligning central nuclei-containing (DAPI, blue), regenerating myofibers in interstitial spaces of the TTDN-operated group at 6 weeks after TTDN (B, white arrows, and $\mathbf{H}$; red arrow), clustered in the perimysium (C, arrow) and occupying interstitial spaces of sham-operated RC (C, $\mathbf{F}$, and $\mathbf{J}$ ), aligning ( $\mathbf{D}$ and $\mathbf{E}$, higher magnification, red arrow) and in proximity (L, red arrow) to $\alpha-S M A^{+}$perivascular cells in injured RC, and localizing fibro-adipogenic lesions ( $\mathbf{G}$ and $\mathbf{H}$, higher magnification, white arrow; and $\mathbf{K}$ ). $\alpha$-SMA (C-L, bright green) is highly expressed by perivascular cells in sham- and TTDN-operated RC (white arrows) but not coexpressed by engrafted CM-Dil+ LR-PC. Asterisk marks myofibers containing DAPI+ central nuclei. A, adipocyte. Scale bars: $50 \mu \mathrm{m}$.

which defines pro-fibro-adipogenic precursors in muscle tissue $(10,12,26)$, revealed a lack of expression of both markers by hES PCs between passages 2 and 9 (Figure 2A), implying that these cells are devoid of fibro-adipogenic features. In accordance, CD146 + PDGFR $-\beta^{+}$PDGFR- $\alpha^{-}$hES PCs failed to differentiate into adipocytes in adipogenic culture (Figure 2, D and E) and similarly to non-induced PC (Figure 2F) did not take up the Oil Red O lipid dye (Figure 2G). Expanded hES PCs resisted TGF- $\beta 1$-induced fibrosis even after 6 weeks of stimulation, as indicated by unaltered levels of collagen synthesis in fibrotic conditions and lack of expression of $\alpha$-smooth muscle actin ( $\alpha$-SMA), a myofibroblast marker (Figure 2, H-K). Additionally, expanded hES PCs did not express CD56 (Figure 2C), which demarcates human myogenic perivascular cells (14), and in accordance rarely formed myotubes in myogenic culture conditions (data not shown). hES PCs were not osteogenic either, as assessed by absence of alizarin red staining in osteogenic 
Table 1. Distribution of LR-PCs engrafted in RC

\begin{tabular}{|c|c|c|c|c|c|c|}
\hline & \multicolumn{3}{|c|}{ Sham } & \multicolumn{3}{|c|}{ TTDN } \\
\hline $\begin{array}{c}\text { LR-PC } \\
\text { differentiation } \\
\text { index }\end{array}$ & $\begin{array}{l}4 \text { days after } \\
\text { injection }\end{array}$ & $\begin{array}{l}2 \text { weeks after } \\
\text { injection }\end{array}$ & $\begin{array}{l}6 \text { weeks after } \\
\text { injection }\end{array}$ & $\begin{array}{l}4 \text { days after } \\
\text { injection }\end{array}$ & $\begin{array}{l}2 \text { weeks after } \\
\text { injection }\end{array}$ & $\begin{array}{l}6 \text { weeks after } \\
\text { injection }\end{array}$ \\
\hline Fibrotic & 0 & 0 & 0 & 0 & 0 & 0 \\
\hline Adipogenic & 0 & 0 & $0.1 \pm 0.5$ & 0 & 0 & 0 \\
\hline Myogenic & $0.1 \pm 0.3$ & $0.2 \pm 0.4$ & $0.05 \pm 0.2$ & $0.2 \pm 0.5$ & $0.5 \pm 1$ & $0.2 \pm 0.4$ \\
\hline $\begin{array}{c}\text { Fibro-adipogenic } \\
\text { scar }\end{array}$ & 0 & 0 & 0 & $3.5 \pm 1.3$ & $9.3 \pm 0.8^{A}$ & $5.3 \pm 1.5$ \\
\hline
\end{tabular}

CM-Dil-labeled LR-PCs were injected into sham-operated or injured RC at the indicated time points. CM-Dil+DAPI+ human LR-PC were identified and counted in multiple sections of engrafted RC at 6 weeks after injury (LR-PC injections at 4 days and 2 weeks after injury) and 10 weeks after injury (LR-PC injection

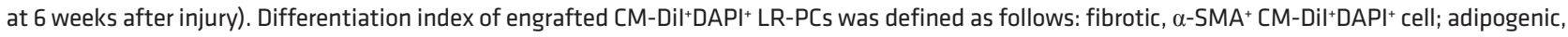
$\mathrm{CM}-\mathrm{Dil}^{+} \mathrm{DAPI}{ }^{+}$adipocyte; and myogenic, myofiber-fused CM-Dil+DAPI+ cell. Data are expressed as mean $\pm \mathrm{SEM} . n=4$ mice per group. ${ }^{A} P<0.05$ (1-way ANOVA).

cultures (Figure 2, L and M). Altogether, these findings suggest that prolonged exposure of hES PCs to growth factors and components that support endothelial cell proliferation preserve the non-fibro-adipogenic, non-osteogenic features of these cells. Characteristics of hES-derived LR-PCs were compared with those of counterpart cells derived from human skeletal muscles (deltoid and subscapularis). Flow cytometry analysis revealed that application of growth factor-enriched endothelial cell growth medium to muscle explant cultures resulted in the expansion of $\mathrm{CD} 146^{+}$cells composed of 2 subpopulations: $\mathrm{CD} 146^{+} \mathrm{CD} 56^{+}$cells and CD $146^{+} \mathrm{CD} 56^{-}$cells (Figure $2 \mathrm{~N}$ ). Only the PDGFR $-\beta^{+} \mathrm{CD} 146^{+} \mathrm{CD} 56^{-}$cell subset, but not CD146 ${ }^{+} \mathrm{CD} 56$ cells (data not shown), coexpressed PDGFR- $\alpha$ (Figure 2N). Coinciding with published data $(12,27,28)$, sorted and expanded PDGFR- $\alpha^{+}$CD56 cells lacked myogenic potential (Figure 2O) in comparison with the myogenic PDGFR- $\alpha$ CD56 ${ }^{+}$cell population that efficiently generated multinucleated myosin heavy chain-positive $\left(\mathrm{MyHC}^{+}\right)$myotubes in identical culture conditions (Figure 2P). Additionally, expression of PDGFR- $\alpha$ was directly correlated with a high adipogenic potential of PDGFR- $\alpha^{+} \mathrm{CD} 56^{-}$cells (Figure $2 \mathrm{Q}$ ), whereas only few adipocytes were detected in PDGFR- $\alpha \mathrm{CD} 56^{+}$adipogenic cultures (Figure $2 \mathrm{R}$ ). As a confirmation of high fibrogenic potential, collagen production was rapidly and significantly upregulated within 4 days in cultures of PDGFR- $\alpha^{+} \mathrm{CD} 56^{-}$cells that were stimulated with the profibrogenic cytokine TGF- $\beta 1$ (Figure 2S). These findings clearly demonstrate that application of endothelial factor-enriched medium does not change the intrinsic characteristics of skeletal muscle tissue-derived cell counterparts, either non-myogenic, fibro-adipogenic PDGFR- $\alpha^{+}$CD56- cells (FAPs) or myogenic PDGFR- $\alpha{ }^{-}{ }^{-}{ }^{-} 56^{+}$cells, and supports the notion that this set of growth factors determine cell fate at early stages of progenitor specification from pluripotent stem cells.

Transplanted LR-PCs maintain non-fibro-adipogenic features. Lack of fibro-adipogenic differentiation properties implies that LR-PCs will be superior for the cell therapy of the chronically injured RC, regenerating muscle and not contributing to degenerative remodeling. To test this hypothesis, CM-DiI-labeled human LR-PCs were administered to chronically injured RC muscles of immunodeficient NOD/SCID mice. LR-PCs were injected at different time points corresponding with stage-specific remodeling of the RC after injury: (a) pro-fibro-adipogenesis stage at 5 days after TTDN, (b) intermediate stage of fibro-adipogenesis at 2 weeks after TTDN, and (c) end-stage fibro-adipogenesis at 6 weeks after TTDN (Figure 3A). Matched controls included cell injection into sham-operated RC and saline- and FAP-injected TTDN $\mathrm{RC}$ at 5 days, 2 weeks, and 6 weeks after surgery (Figure 3A). At 4 weeks after injection, CM-DiI ${ }^{+}$human cells were still detected in muscle interstitial spaces in proximity to myotubes of injured (Figure 3, B and D) or sham-operated RC (Figure 3, C, F, and J). Furthermore, human cells were incorporated in the fibrotic scar in end-stage fibro-adipocytic muscles (at 6 and 10 weeks after TTDN) in all tested groups (Figure $3, \mathrm{G}, \mathrm{H}, \mathrm{I}, \mathrm{K}$, and L). $\alpha$-SMA is a marker of perivascular smooth muscle cells and myofibroblasts. Immunostaining of RC sections with cross-reactive anti-mouse and -human $\alpha$-SMA antibodies demonstrated high $\alpha$-SMA expression in blood vessel-residing cells (Figure 3, C-L) but not in engrafted CM-DiI ${ }^{+}$cells in all sham and TTDN groups (Table 1), implying that transplanted LR-PCs do not transdifferentiate 

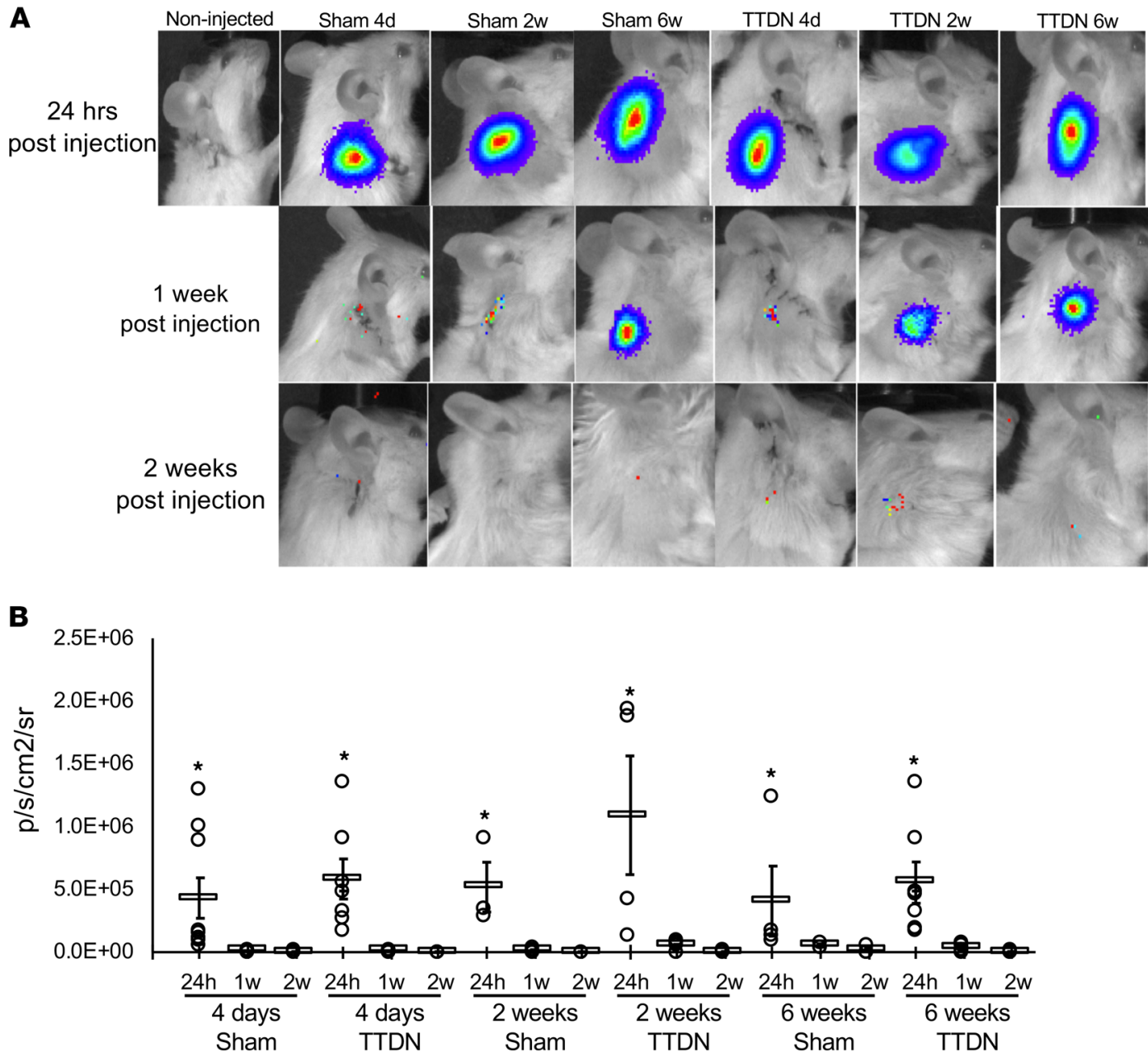

Figure 4. Bioluminescence imaging of luciferase-positive injected LR-PCs. (A and B) High signal was recorded at 24 hours, drastically declined within 1 week, and disappeared at 2 weeks after injection of luciferase-labeled LR-PCs into sham- or TTDN-operated RC at the indicated time points after surgery. (A) Representative optical images of luciferase activity of injected LR-PCs at the indicated type of surgery, time point of cell injection, and timing of in vivo imaging. sr, steradian. (B) Quantification of luciferase signal. Data are expressed as mean \pm SEM, $n=$ at least 3 mice per group, and ${ }^{*} P<0.05$ compared with matched TTDN or sham surgeries at 1-week and 2-week injection time points (1-way ANOVA).

into myofibroblasts, either spontaneously or in response to fibrotic cues. We then evaluated whether the restricted adipogenic differentiation of cultured LR-PCs is stimulated when injected into sham-operated and injured RC, through quantification of $\mathrm{CM}$-DiI ${ }^{+}$adipocyte progeny. Except for a few rare CM-DiI ${ }^{+}$ adipocytes that were detected when LR-PCs were injected at the end stage of RC degeneration, at 6 weeks after TTDN, LR-PCs were devoid of adipogenic potential in vivo (Figure 3G and Table 1), suggesting that engrafted cells were still unable to respond to prolonged environmental adipogenic cues. Finally, few CM-DiI ${ }^{+}$LR-PCs were detected fused to murine myofibers, independent of injection timing in all tested groups (Table 1).

To evaluate the survival of implanted cells, CM-DiI ${ }^{+}$LR-PCs were stably transduced to express luciferase, and bioluminescence intensity was monitored at days $1,7,14,21$, and 28 , using a luciferase-based live imaging system. The strong bioluminescence signal measured in all sham-operated and TTDN-injected groups at day 1 had significantly declined by day 7 and further diminished within 2 weeks (Figure 4, A and B). It has been previously shown that with time, a sharp drop in bioluminescence can be followed by escalating measurements, indicative of selective cell proliferation or potential cell tumorigenesis (29). The luciferase-LR-PC signal was not detected beyond 2 weeks after injection in all tested groups (Figure 4B). However, CM-DiI ${ }^{+}$LR-PCs were still detected in muscle interstitial spaces of sham-operated and TTDN RC at 4 weeks (Figure 3A, groups B and C) and up to 6 weeks (Figure 3A, group A) after surgery (Figure 3C and Table 1), suggesting that signal loss can be partially attributed to cell migration away from the injection site. 

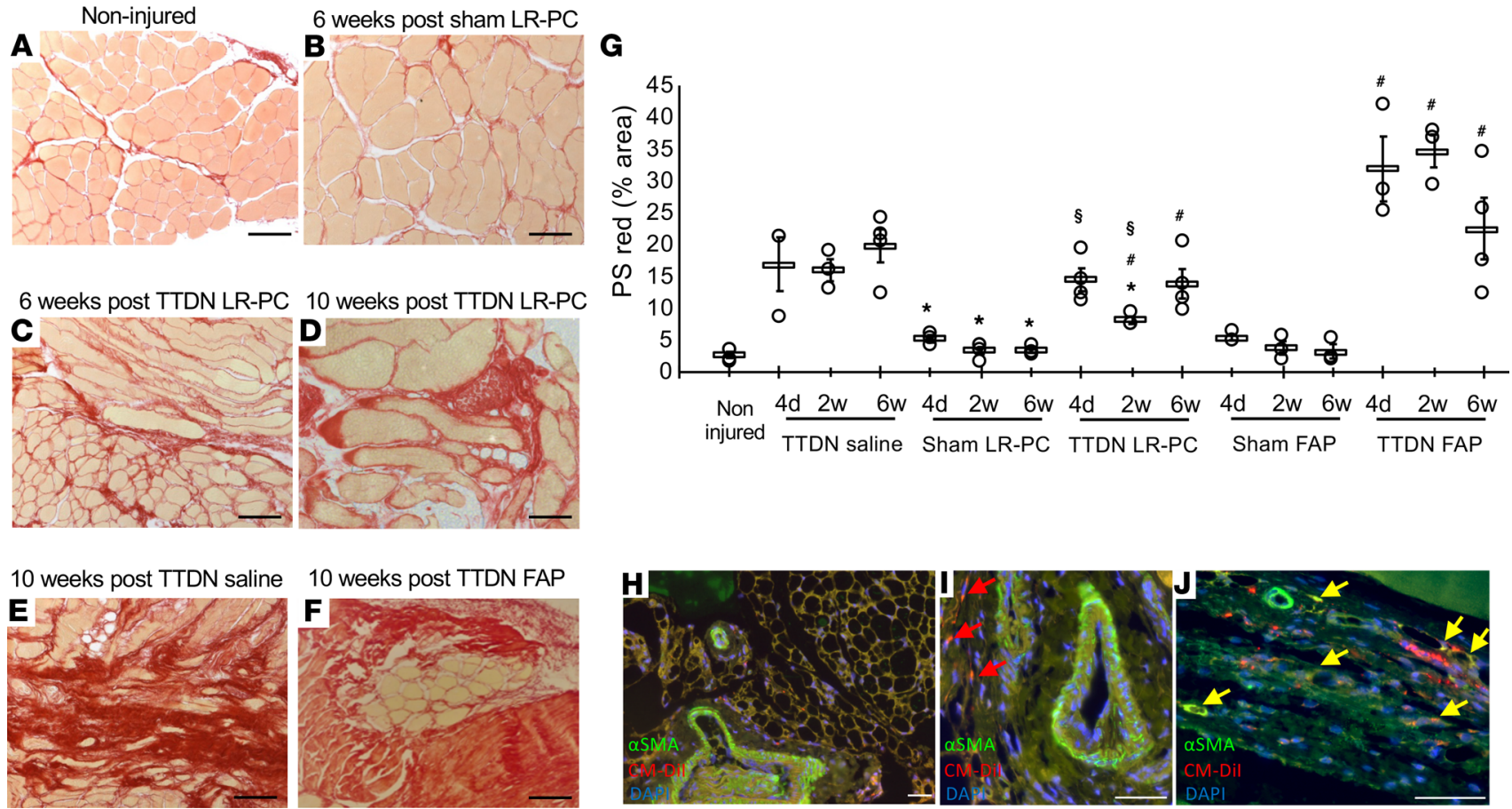

Figure 5. Transplanted LR-PCs but not FAPs diminish the development of fibrosis in chronically injured RC. (A-F) Representative images of picrosirius red staining for collagen of RC sections at the indicated settings. (G) Red pixel intensity quantification of picrosirius red-stained sections of non-injured, sham-operated, and injured RC at 6 or 10 weeks after operation that were injected with saline, LR-PCs, or FAPs at the indicated time points. LR-PCs significantly inhibited the development of fibrosis in injured muscle when injected at 2 and 6 weeks after TTDN. FAPs significantly increased fibrosis at all time points of injection. Data are expressed as mean $\pm \mathrm{SEM} ; n=3-4$ mice per group, 5 picrosirius red-stained regions per RC section. ${ }^{*} P<0.05$ compared with matched time points of saline injections after TTDN. ${ }^{\#} P<0.005$ between cell injected TTDN mice and matched sham cell injected mice. ${ }^{\S} P<0.05$ between LR-PC-injected TTDN mice and matched FAP-injected TTDN mice (1-way ANOVA). (H-J) Transplanted CM-Dil-labeled LR-PCs (H and I, red) did not contribute

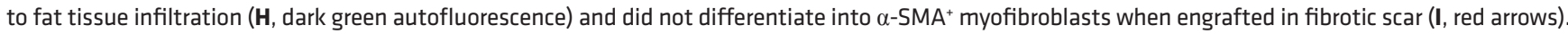
$\alpha$-SMA (green) was expressed by perivascular cells of large blood vessels (H-J) and engrafted FAPs (yellow arrows, J). Scale bars: $100 \mu \mathrm{m}(\mathbf{A}-\mathbf{F}), 50 \mu \mathrm{m}(\mathbf{H}-\mathbf{J})$.

Quantification of CM-DiI ${ }^{+}$cell deposition within remodeling injured RCs revealed that recruitment of implanted cells into fibro-adipogenic scar tissue was significantly increased when cells were injected at 2 weeks after TTDN $(P<0.05)$ in comparison with cell injection at 4 days and 6 weeks after injury (Table 1).

$L R-P C$ s inhibit fibrosis but not adipogenesis in the injured $R C$. We next measured collagen accumulation, as a key mediator of muscle fibrosis, by picrosirius red staining and red pixel intensity quantification. As expected, a progressive increase in collagen production was observed up to 10 weeks after TTDN in all saline-, LR-PC- and human muscle FAP-injected groups A-C, regardless of the timing of injection (Figure 5, A-F). However, LR-PC injections tended to reduce collagen accumulation (Figure 5G), and a significant 2-fold reduction in fibrosis was measured when cells were injected at 2 weeks after TTDN, implying that LR-PCs exert the best prevention of fibrosis when administered at an earlier stage of muscle degeneration. In accordance, a significant increase in engraftment of LR-PCs within the developing fibro-adipogenic scar was only detected when cells were injected at 2 weeks after TTDN (Table 1). Additionally, engrafted LR-PCs were entirely devoid of expression of the myofibroblast marker $\alpha$-SMA, implying that LR-PCs did not contribute to the development of fibrosis when injected into sham- or TTDN-operated RC (Figure 5, H and I, and Table 1). Altogether, these findings strongly support the notion that LR-PCs maintain non-fibrotic differentiation potential in the healthy and fibrotic muscle. Human skeletal muscle-derived, PDGFR- $\alpha^{+}$CD56- FAPs were used as compatible controls of transplantation experiments, and the effect of matched administered FAPs on the development of fibrosis was evaluated (Figure 5, F, G, and J). Significantly greater fibrosis was measured when FAPs were administered at early, (4 days), intermediate, (2 weeks) and late stages (6 weeks) of muscle degeneration (Figure 5G). Similar to LR-PCs, engrafted CM-DiI ${ }^{+}$FAPs could still be observed in sham and injured RC sections at 4 weeks after injection, but unlike LR-PCs, FAPs accumulated at the injection site and expressed $\alpha$-SMA in injured muscle (Figure $5 \mathrm{~J}$ ). 


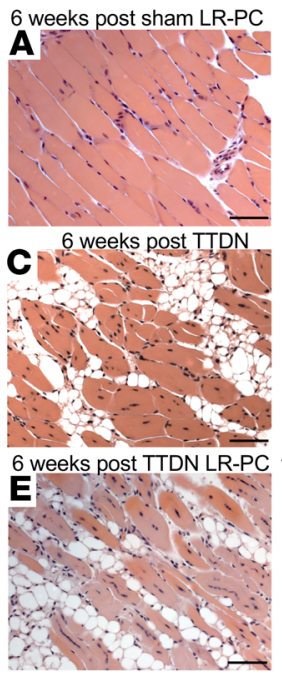

6 weeks post TTDN FAP

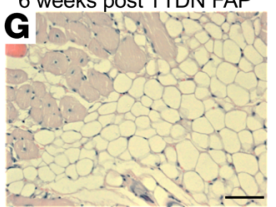

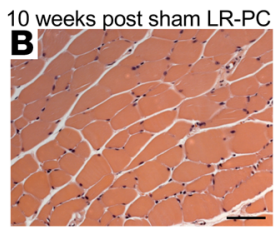

10 weeks post TTDN
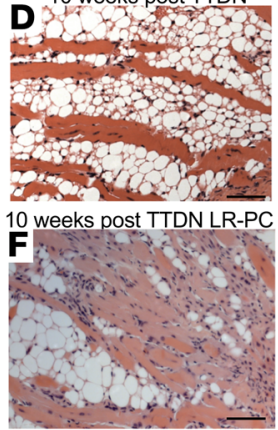

10 weeks post TTDN FAP

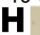

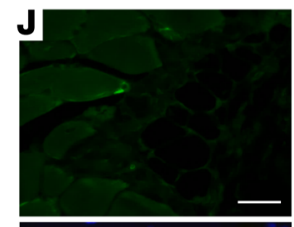

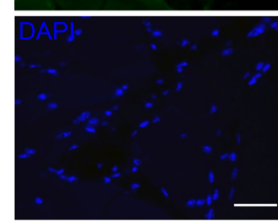

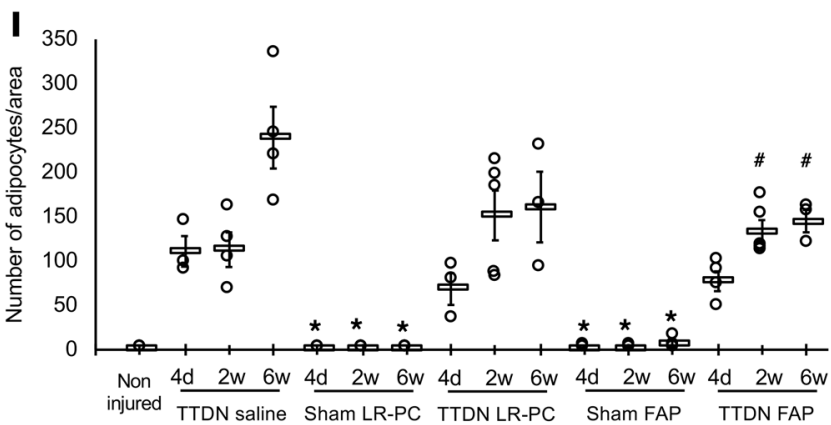

injured $\overline{\text { TTDN saline Sham LR-PC TTDN LR-PC }} \overline{\text { Sham FAP }} \overline{\text { TTDN FAP }}$

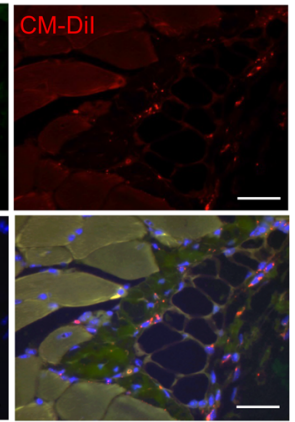

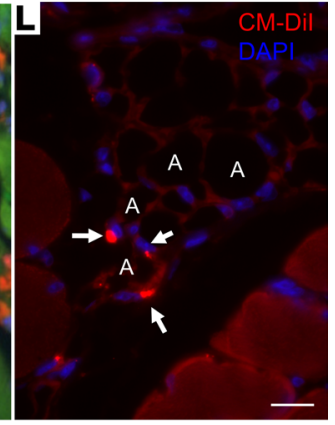

Figure 6. Transplanted LR-PCs maintained non-adipogenic properties and did not affect adipocyte content in chronically injured RC. H\&E staining of RC sections demonstrated that injured muscle undergoes prominent morphological change from fat-free tissue characteristic of non-injured (A) or sham-operated muscle at 10 weeks after surgery $(\mathbf{B})$ to atrophied muscle with robust fatty infiltration in non-injected (C and $\mathbf{D})$, LR-PC-injected (E and $\mathbf{F}$ ), and FAP-injected ( $\mathbf{G}$ and $\mathbf{H}) \mathrm{RC}$ at 6 weeks (C, E, and $\mathbf{G}$ ) and 10 weeks (D, F, and $\mathbf{H})$ after TTDN. (I) Quantification of adipocyte numbers in H\&Estained RC tissue sections. Data are expressed as mean \pm SEM; $n=3-5$ mice per group, 5 adipocyte containing regions per RC section. ${ }^{*} P<0.005$ sham LR-PC- or FAP-injected mice in comparison with matched time points of saline or cell injections after TTDN. \# $P<0.05$ compared with FAP injected mice at 4 days after TTDN (1-way ANOVA). (J and K) CM-Dil (red) labels engrafted LR-PC in interstitial spaces but not fat tissue-repopulating adipocytes (K); white arrows indicate CM-Dil- adipocytes). (L) FAP-derived CM-Dil ${ }^{+}$adipocytes (arrows) in injured RC. (Blue nuclear staining for DAPI.) A, adipocyte. Scale bars: $100 \mu \mathrm{m}(\mathbf{A}-\mathbf{H}), 50 \mu \mathrm{m}(\mathbf{J})$, and $20 \mu \mathrm{m}$ (K and $\mathbf{L})$

We next imaged areas of fatty infiltration in all tested groups $(\mathrm{A}-\mathrm{C})$ for quantification of adipocytes in RC sections stained with H\&E (Figure 6, A-H). Administration of LR-PCs did not induce (Figure 6I) or directly contribute to muscle adipogenesis (Figure 6, J and $\mathrm{K}$, and Table 1). Except for a trend toward reduction of adipocytes when injected 6 weeks after TTDN, LR-PCs did not inhibit adipogenesis at any other given time point of cell injection (Figure 6I). FAP administration (Figure 6H) did not affect the rate of fat accumulation in injured muscle. Overall, FAPs remained clustered at the injection site and were not recruited to regions of atrophied muscle, but $\mathrm{CM}_{-} \mathrm{DiI}{ }^{+} \mathrm{FAPs}$ were still induced to differentiate into adipocytes at all time points of injection into injured fibro-adipogenic muscle (Figure 6L).

LR-PCs attenuate chronic muscle atrophy. Atrophy of the torn RC is concurrent with muscle degeneration and can be recognized on injured muscle sections by the reduction in the diameter of myofibers. The average myofiber cross section diameter in non-injured RC muscle was $80 \pm 2.7 \mu \mathrm{m}$ ( $n=3$ mice, at least 15 sections per mouse, total of 83 myofibers) and was set as the threshold diameter of non-atrophic myofibers. Although peripheral nuclei generally indicate healthy, mature myofibers, nuclei central to myofibers reflect regeneration, even though they are also encountered in centronuclear myopathies involving muscle denervation (29-31). Accordingly, we classified and quantified the following distinct types of myofibers: (a) non-atrophic, with central nuclei; (b) non-atrophic, with peripheral nuclei; (c) atrophic, with central nuclei; and (d) atrophic, with peripheral nuclei. As expected, TTDN injury induced massive myofiber atrophy $(70 \%-90 \%)$ in comparison with sham-operated controls within 6-10 weeks (Figure 7, A, B, D, and G). Of note, similar degrees of atrophy were apparent in both types of myofibers, peripheral $(\mathrm{M})$ and centronuclear (CNM), indicative of accumulative muscle wasting and cycles of ineffective muscle regeneration (Figure 7G). The operation of LR-PC injection itself slightly damaged the muscle, inducing approximately $20 \%-30 \%$ of atrophy in both types of myofibers in all sham control groups, but this was nearly 4-fold less than TTDN-induced atrophy (Figure 7, A, B, and G). 

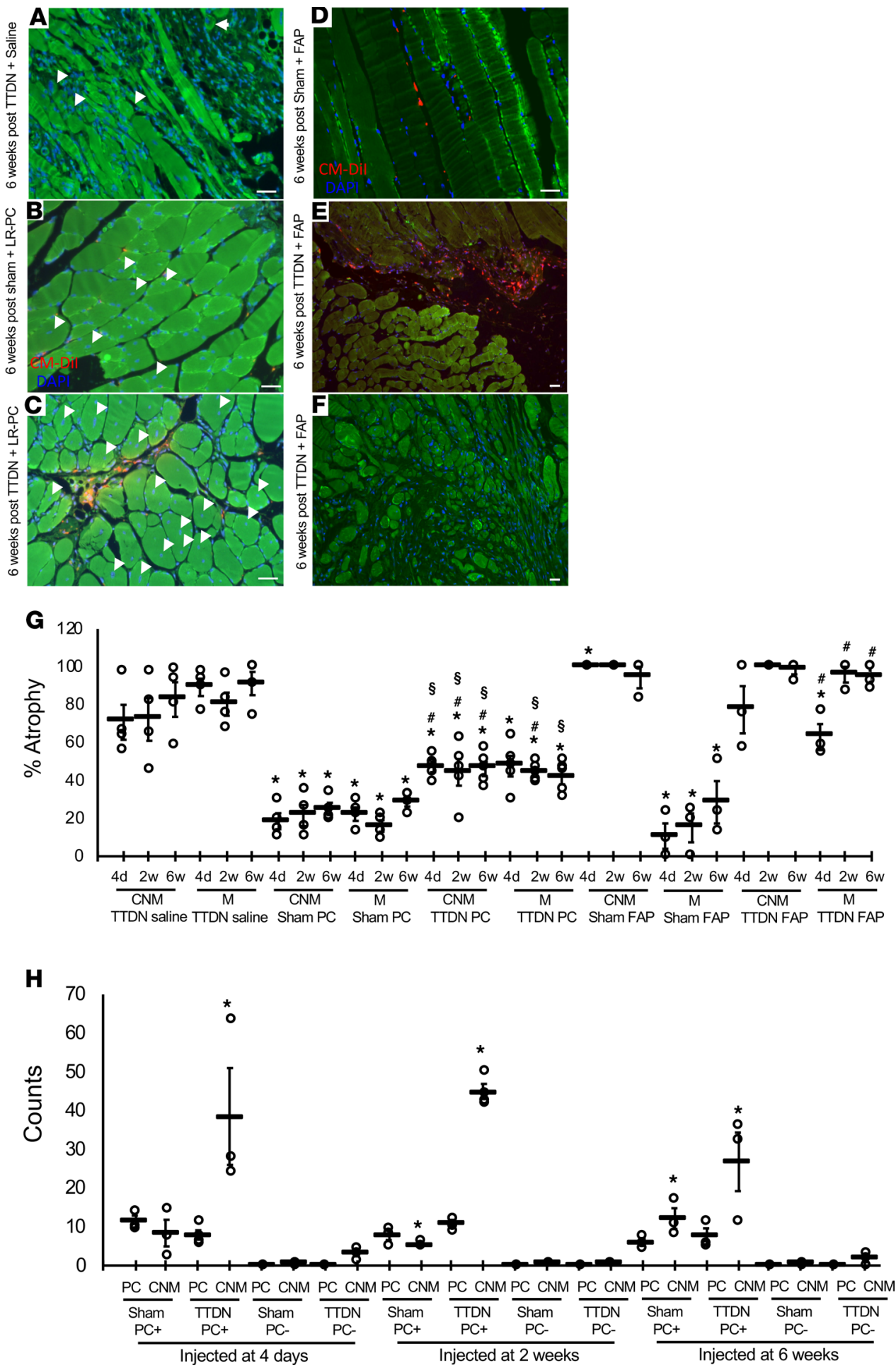

Figure 7. Transplanted LR-PCs but not FAPs attenuate RC muscle atrophy. (A) TTDN injury induced severe muscle wasting and atrophy within 6 weeks. Myofibers with significantly decreased diameter are indicated by arrows. (B) Injection of CM-Dil+ LR-PC (red) resulted in minor regeneration of non-atrophied myofibers in proximity to the site of injection as indicated by centrally located DAPI ${ }^{+}$ nuclei (blue, arrows). (C) Injection of CM-Dil+ LR-PCs resulted in significant inhibition of myofiber atrophy of central nuclei-regenerating (blue, arrows) myofibers at 6 weeks after TTDN. (D) Injected CM-Dil ${ }^{+}$FAPs were localized in interstitial spaces between healthy myofibers ( $\mathbf{E}$ and $\mathbf{F}$ ). CM-Dil ${ }^{+}$FAPs in injured RC remained clustered $(\mathbf{E})$ and did not migrate to regions of muscle atrophy (F) at 6 weeks after TTDN. (C) Cross section measurements of CNMs and peripheral nuclei myofibers (M) of sham- or TTDN-operated $\mathrm{RC}$ injected with CM-Dil+ $\mathrm{LR}-\mathrm{PC}$ at the indicated time points. Matched injections of saline into TTDN-operated RC served as muscle atrophy reference. Data are expressed as mean \pm SEM. $n=3-5$ mice per group, ${ }^{*} P<0.05$ compared with matched time saline-injected TTDN mice, ${ }^{\#} P<0.05$ compared with matched sham-operated mice, and $\$ P<0.05$ between LR-PC- and matched FAPinjected TTDN mice (1-way ANOVA). Scale bars: $50 \mu \mathrm{m}$. (H) Frequency of central nuclei myofibers (CNMs) and transplanted $\mathrm{PC}$ in tissue sections of injected RC. PC+, LR-PC engrafted regions in tissue sections of injected RC; PC-, LR-PC non-engrafted regions in tissue sections of injected RC. $n=4$ mice per group and 5 LR-PC-containing regions per RC section. Data are expressed as mean \pm SEM. $P<0.05$ between CNMs in $\mathrm{PC}^{+}$regions and $\mathrm{CNM}$ in matched $\mathrm{PC}^{-}$regions (1-way ANOVA).

LR-PCs did not differentiate into myotubes after injection (Table 1) but significantly diminished muscle atrophy at all time points of injection (4 days, 2 weeks, and 6 weeks after TTDN), when compared with LR-PC-matched sham controls and saline-injected TTDN-injured mice (Figure 7, C and G). To map the anatomical sites of LR-PC engraftment, we quantified atrophied centronuclear myofibers (indicative of muscle loss) in 2 distinct areas within the same tissue section: (a) area in which CM-DiI ${ }^{+}$ LR-PC engraftment was detectable and (b) area in which CM-DiI ${ }^{+}$LR-PC engraftment was undetectable. LR-PCs were predominantly distributed in areas of degenerating muscle as indicated by a significantly greater number of atrophic CNMs in engrafted areas in comparison with non-engrafted areas. 

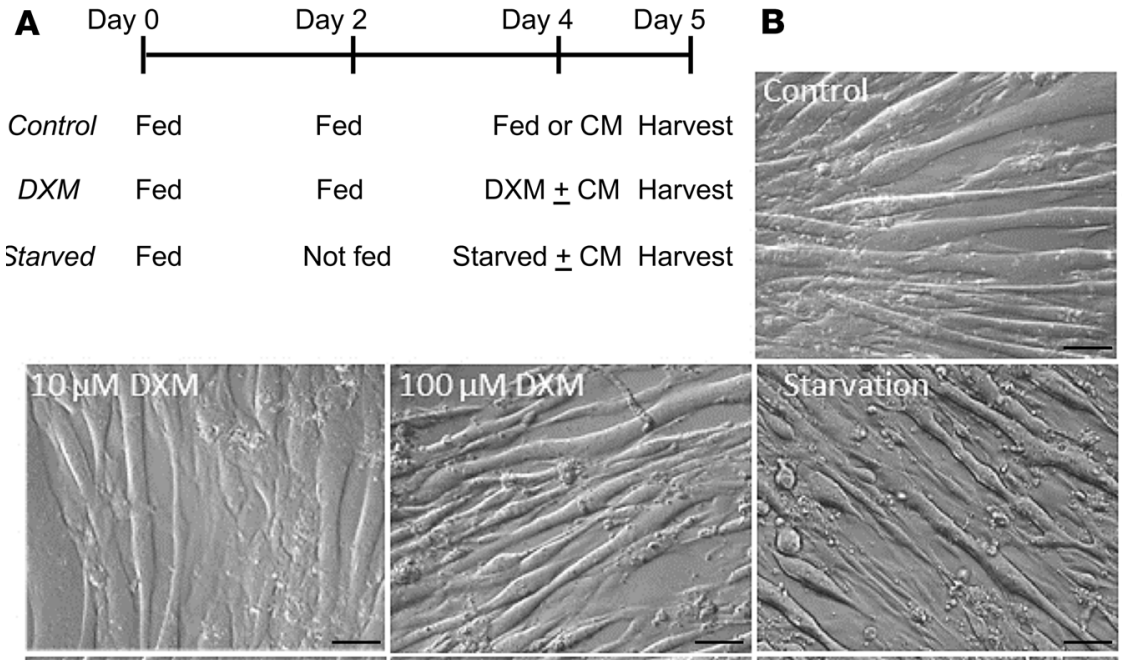

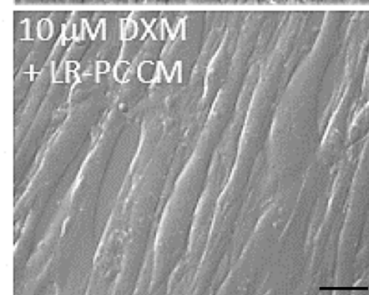

C

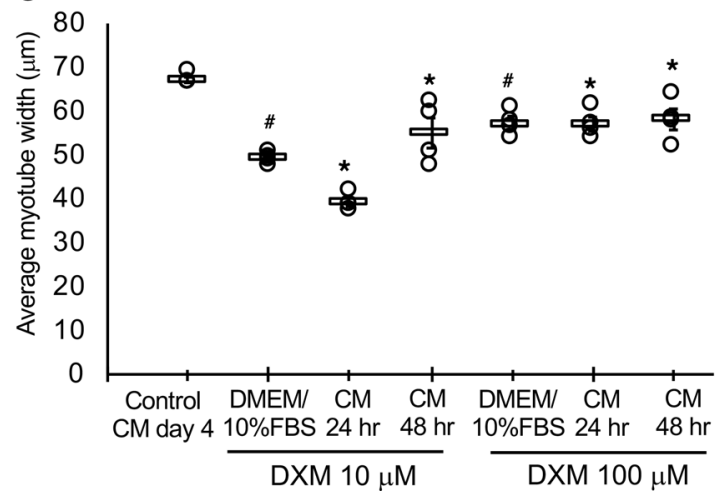

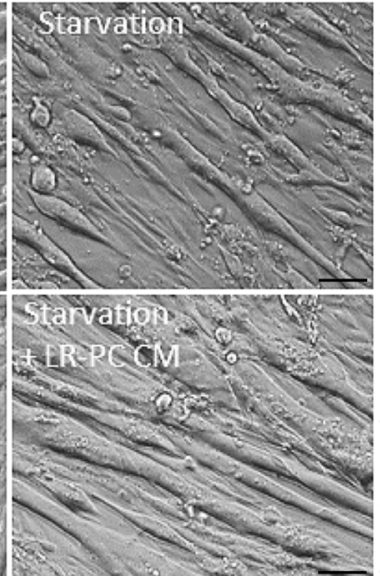

D

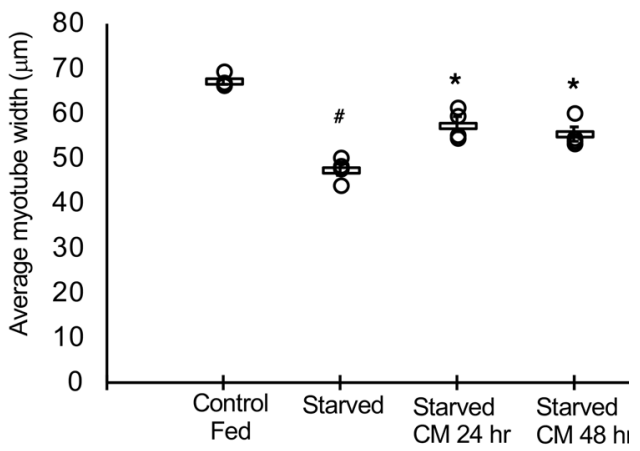

Figure 8. LR-PC-conditioned medium rescues myofiber atrophy. (A) Schematic illustration of the in vitro atrophy models and experimental design. DXM, dexamethasone; CM, conditioned medium. (B) Representative images of morphological changes of cultured C2C12 murine myotubes. Cultured myotubes were treated with $10 \mu \mathrm{M}$ or $100 \mu \mathrm{M}$ DXM for 24 hours or starved for induction of myofiber atrophy in the presence or absence of LR-PC-derived CM. (C and D) Quantification of average myotube width at the indicated conditions demonstrates that LR-PC CM attenuates both DXM- (C) or starvation-induced (D) atrophy of cultured myotubes. CM 24 hr, CM was collected at 24 hours; CM 48 hr, CM was collected at 48 hours. DMEM/10\% FBS was used for DXM-induced atrophy and as feeding and control medium for starvation-induced atrophy. Data are expressed as mean \pm SEM. ${ }^{*} P<0.0005$ between DXM-treated and DXM CM-treated myofibers or between starved and starved CM-treated myotubes, and $P<0.0005$ between controls and DXM-treated or -starved myotubes (1-way ANOVA). $n=4$ independent experiments, $n=20-25$ myotubes per control group, $n=65-150$ myotubes per DXM groups, and $n=70-180$ myotubes per starved groups. Scale bars: $100 \mu \mathrm{m}$.

This trend was observed in all treated groups $(\mathrm{A}-\mathrm{C})$, with significantly more atrophic CNMs in the

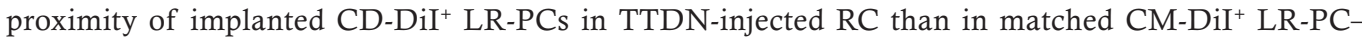
injected, sham-operated RC (Figure 7H). Conversely, injected FAPs were detected in interstitial spaces only in sham-operated RC (Figure 7D) and were clustered at the injection site in injured muscle (Figure 7E). Additionally, FAPs were not recruited to regions of muscle atrophy regardless of time point of injection after TTDN (Figure 7F). Altogether, these findings demonstrate that non-fibro-adipogenic transplanted PCs are selectively recruited to sites of muscle damage where they attenuate chronic muscle atrophy. 
$L R$-PC culture-conditioned medium rescues atrophied myotubes. Absence of myogenic differentiation by engrafted LR-PCs suggests that these PCs prevent myofiber atrophy via secretion of factors. To test this hypothesis, we first induced atrophy of cultured murine $\mathrm{C} 2 \mathrm{C} 12$ myotubes by starvation (myotube cultures that were not refed) or treatment with dexamethasone $(32,33)$. LR-PCs were mitotically inactivated and maintained in growth factor-deprived DMEM supplemented with 10\% FBS. Conditioned medium was collected after 24 and 48 hours and assessed for its ability to block dexamethasone- or starvation-induced atrophy of murine C2C12 myoblasts that were induced to fully fuse into myotubes for 3 days in culture (Figure 8A). Controls consisted of myotubes incubated with DMEM/10\% FBS or matched conditioned medium for 24 hours. Myotube average width was not changed under these conditions, demonstrating that LR-PC-conditioned medium alone does not induce myotube atrophy or hypertrophy (Figure 8, B and C). As was previously shown $(32,33)$, addition of dexamethasone resulted in myotube atrophy with a significant dose-dependent decrease $(P<0.0005)$ in myotube average width (Figure 8, B and C). Similarly, myotubes starved 4 days were substantially narrower $(P<0.0005)$ than control fed myotubes (Figure $8, \mathrm{~B}$ and $\mathrm{D})$. Conditioned medium from 24-hour LR-PC cultures effectively blocked dexamethasone- or starvation-induced atrophy within 24 hours. Conditioned medium collected after 48 hours was not more inhibitory of myofiber atrophy, implying that optimal concentration of active factors was reached within 24 hours (Figure 8, C and D).

\section{Discussion}

Degenerative remodeling of injured muscle alters the cellular and extracellular composition of the supportive stroma and consequently leads to severe loss of functional myofibers. Additionally, robust development of adipose and fibrotic tissue, typically as a result of chronic muscle injury, often hinders successful engraftment of functional progenitors. We have recently demonstrated that fibro-adipogenic perivascular cells are typified by coexpression of PDGFR- $\beta$ and PDGFR- $\alpha$ in mouse skeletal muscle $(12,13)$. We decided to extend and validate these findings in human cells. With this aim, we generated a potentially novel line of lineage-restricted cells (LR-PCs) from $h E S$, characterized as PDGFR- $\beta^{+}$PDGFR- $\alpha^{-}$, which defines non-fibro-adipogenic pericyte-like cells, similar to their murine counterparts. Non-fibro-adipogenic characteristics of LR-PCs were preserved in long-term culture as well as the expression of other PC markers, such as CD146 and CD73. Not only did LR-PCs maintain non-fibro-adipogenic characteristics after transplantation into the highly fibro-adipogenic environment of the chronically injured muscle, but they also attenuated RC muscle atrophy and fibrosis through paracrine factors.

Myogenic cells may be therapeutic in the chronically injured RC. However, fibrosis in diseased organs often disrupts efficient engraftment of functional progenitors due to a hostile microenvironment. For instance, the myogenicity of satellite cells may be compromised in the fibrotic muscle (34). Therefore, it is important for the development of improved stem cell-based therapies to distinguish FAPs from regenerative stromal cells for successful reconstruction of the deteriorating stromal niche. PDGFR- $\alpha$-expressing, muscle-resident perivascular and interstitial stromal cells have been identified as FAPs, the presence of which is correlated with the development of fibrosis and fat accumulation in mouse muscle after acute $(10,12)$ or chronic $(13)$ injury, as well as the severity of fibrosis in patients with Duchenne muscular dystrophy (27). Besides being a useful fibro-adipogenic cell marker, the signaling receptor PDGFR- $\alpha$ functionally regulates fibrogenic responses associated with multiple cardiac, renal, and muscle pathologies. PDGFR- $\alpha$ is activated predominantly in FAP cells in regenerating muscle, mediating ERK1/2 and AKT downstream signaling (35). Additionally, elevated PDGFR- $\alpha$ signaling in embryos leads to accelerated fibrosis and disruption of connective tissue development (36). We demonstrate here that hES PCs uniformly express PDGFR- $\beta$, which has been implicated as a marker of fibro-adipogenesis as well. Conversely, PDGFR- $\beta$ typifies mouse and human mesodermal perivascular precursors that exhibit long-term multilineage differentiation capability, including in the myogenic cell lineage $(9$, $15,37)$, indicating functional heterogeneity of PDGFR- $\beta^{+}$cells and strongly suggesting that coexpression of PDGFR- $\alpha$ by PDGFR- $\beta$ cells is a key determinant for identification of PDGFR- $\beta^{+}$FAP cells. Supporting this definition, severe cardiac fibrosis was detected in PDGF- $\alpha$ - but not PDGF- $\beta$-overexpressing transgenic mice (38), and we have recently demonstrated in PDGFR- $\beta$ reporter mice that GFP PDGFR- $\beta^{+}$cells that coexpress PDGFR- $\alpha$ directly contribute to fibrotic degeneration in acutely (12) and chronically (13) injured skeletal muscle. In accordance, we demonstrate here that classification of mouse FAPs based on coexpression of PDGFR- $\beta$ and PDGFR- $\alpha$ is also applicable for human cells, in the context of skeletal muscle wasting. PDGFR- $\beta / P D G-$ FR- $\alpha$-based identification and classification should be further validated by additional analysis of PDGFR- $\beta$ / PDGFR- $\alpha$ expression on human cells from multiple organs with functional differentiation assays. 
Stemming of fibrosis and fat accumulation at any tested time point of LR-PC transplantation after muscle injury is extremely promising because a potential complication with usual regenerative cell injections is that these may follow a profibrotic pathway when no longer exposed to culture conditions that inhibit their differentiation potential or may secrete factors that increase the risk of adverse outcomes $(39,40)$. Coinciding with these results, we have previously used the same model of chronically injured muscle to test the therapeutic effect of long-term expanded perivascular cells from human adipose tissue and demonstrated that these cells do not worsen muscle degeneration and indirectly diminish muscle atrophy (8). Differing from human muscle-derived PCs that can adopt myogenic features immediately after purification (15) as well as after long-term culture (9), LR-PCs rarely formed myotubes and only at very early passages (data not shown), indicating a lack of myogenic potential of LR-PCs, which persisted when cells were injected into regenerating muscles at 4 days and 2 weeks after injury. Overall, development of fibrosis involves activation of signaling cascades that induce migration of myofibroblasts to sites of injury. The fibrogenic response is initiated in chronically injured RC at 2 weeks after operation (13), and in accordance we observed a marked increase in the recruitment of transplanted LR-PCs to regions of damaged muscle at 2 weeks after injury, implying stage-specific upregulation of factors that mediate stromal cell migration, such as TGF- $\beta$, PDGF- $\alpha / \beta$, and Ang I (37, 38, 41). Given that exposure of atrophied myotubes to LR-PC-conditioned medium was sufficient to significantly reduce atrophy in culture, further experiments will be carried out to identify and characterize the released therapeutic factors (free secreted growth factors and/or exosomes). Paracrine factors secreted by stromal cells may mediate tissue healing through inhibition of apoptosis, stimulation of cell proliferation, differentiation, modulation of immune-inflammatory reactions, and induction of neovascularization. A pivotal role of endothelial hepatocyte growth factor in the inhibition of lung fibrosis was attributed to suppression of the profibrotic expression of NOX4 in lung and liver perivascular cell-derived fibroblasts (42). PC-derived insulin-like growth factor 1 (IGF-1) was identified as a secreted effector regulating myofiber growth in postnatal mice (43), and injection of IGF-1 into mouse muscle with denervation-induced atrophy mediated a decrease in the expression of the atrophy-related genes $M A F b x$ and $M u r F 1$ and rescued muscle mass (32). Human skeletal muscle PCs express the promyogenic microRNA mir-206 (44), which was shown to regulate skeletal muscle fiber hypertrophy and collagen deposition (45). The results obtained from the in vitro atrophy experiments suggest that muscle atrophy is reduced regardless of anti-fibrotic effects that can be possibly linked to or independent of activation of anti-atrophic responses. Because LR-PC supernatant did not make normal myotubes hypertrophic, a compensatory hypertrophy targeted specifically to atrophied myotubes cannot be excluded.

Multiple protocols to differentiate hPSCs into mesodermal precursors and mesenchymal stem-like cells exist. However, functional differentiation testing demonstrates that in most cases, hPSC mesodermal derivatives are heterogenous cell populations composed of a mixture of mesodermal progenitors with distinct differentiation potentials (e.g., osteo-/chondro-/fibro-/adipogenic) and marker expressions (14). Additionally, modifications take place in long-term cultures, resulting in progressive cell maturation or acquirement of fibroblast-like characteristics. Derivation of non-fibro-adipogenic cells from hPSCs provides a tool for unraveling the molecular mechanisms that determine cell differentiation fate and understanding mechanisms underlying gene expression and identifying novel precursors and sets of markers. Methods such as gene expression profiling of pluripotent stem cells at various stages of induced differentiation (46), RNA-sequencing of murine muscle-derived FAPs at a single-cell level (47), and comparison of human tissue microarray to human pericyte transcriptome (48) were used for comprehensive identification of progenitor subtypes and relevant markers. Similar studies will allow us to characterize a hierarchy of LR-PC precursors and involved molecular control of a non-fibro-adipogenic phenotype. Of note, when endothelial cell growth factor-enriched medium was used to establish cultures of stromal cells from human muscle explants, muscle-derived expanded cells maintained their intrinsic features in short-term and differentiation cultures. As was previously shown $(9,27)$, although not tested at the conditions reported herein, human $\mathrm{CD}^{+} 6^{+}$cells do not express the fibro-adipogenic marker PDGFR- $\alpha$ and in accordance exhibited high myogenic features but no fibro-adipogenic potential, whereas CD56- cells that express PDGFR- $\alpha$ displayed high fibro-adipogenic potential and no myogenic differentiation capability. These features were maintained following transplantation of PDGFR- $\alpha^{+}$FAPs into chronically injured muscles regardless of time point of injection. Our in vitro and in vivo findings clearly demonstrate that application of endothelial factor-enriched medium does not change the intrinsic characteristics of skeletal muscle tissue-derived cell counterparts, supporting the notion that this set of growth factors determine cell fate at early stages of progenitor specification from pluripotent stem cells. 
The use of a novel, simple, and reproducible protocol for generation of non-fibro-adipogenic stromal cells that participate indirectly in the repair of chronically injured RC muscle and do not contribute to degenerative fibro-adipogenic remodeling may have valuable potential in the treatment of multiple muscle disorders. Furthermore, the strategies used in this research may be applicable not only to muscle regeneration but also to microenvironment reconstruction and potential repair in other tissues affected by fibrosis, such as lung, kidney, liver, and heart. Human pluripotent cell-based therapy has already reached the clinical trial stage (49). We envision that this work will result in an autologous therapy that may be used perioperatively to decrease the morbidity of massive RC tears, as well as the failure rate of surgical repairs and resulting disability in millions of people.

\section{Methods}

Mice. NOD/SCID mice (females, 8-10 weeks old) purchased from The Jackson Laboratory (JAX 001303) were used for all experiments.

Cell cultures. hES (H9 cell line) were grown and maintained on hES-qualified Matrigel-coated plates in mTESR medium (Stem Cell Technologies) containing $0.4 \%$ penicillin/streptomycin (Hyclone), as previously described $(46,50)$. All cell lines were tested for mycoplasma and were negative for mycoplasma contamination. Cell line identity was confirmed by karyotype analysis routinely using Cell Line Genetics. Differentiation was initiated when hES reached about 50\% confluence, and hES medium was substituted with EGM-2 medium (Lonza) for 10-14 days. Differentiated adherent cell derivatives were then removed from the culture dish by $0.25 \%$ Trypsin-EDTA (Corning) for multisorting of CD $146^{+} \mathrm{CD} 34^{-} \mathrm{CD} 45^{-}$derivatives. Alternatively, CD $146^{+} \mathrm{CD} 34^{-} \mathrm{CD} 45^{-}$cells dominated the culture within 2 passages when expanded in EGM-2 without the need for cell sorting. Human deltoid and subscapularis were obtained with informed consent $(n=3)$, and muscle stromal cells were expanded in explant cultures supplemented with EGM-2. Confluent adherent cells were then removed (Trypsin-EDTA $0.25 \%$ ) for multisorting of CD $146^{+} \mathrm{CD} 56^{+}$ and $\mathrm{CD} 146^{+} \mathrm{CD} 56^{-}$cells. Sorted subsets were further expanded in EGM-2 for an additional 1-2 passages.

Flow cytometry and cell sorting. Differentiated and expanded hES PCs (passages 2-9) and human muscle cells from primary cultures were washed in PBS, centrifuged, and labeled according to the manufacturer's instructions with the following antibodies: PE-conjugated mouse anti-human PDGFR- $\beta / C D 140 \mathrm{~b}$ (cata$\log 323605)$, PE-conjugated mouse anti-human PDGFR- $\alpha / C D 140$ a (catalog 3235050), APC-conjugated mouse anti-human CD34 (catalog 343509), FITC-conjugated mouse anti-human CD45 (catalog 368508), APC-conjugated mouse anti-human CD56 (catalog 318310), and FITC-conjugated mouse anti-human CD73 (catalog 344015) from BioLegend and PE-conjugated mouse anti-human CD146 from BD Biosciences (catalog 550315). Cell sorting was performed using Aria II Flow Cytometer (BD Biosciences). Debris and dead cells were excluded according to forward and side scatter, and analyses were carried out using LSR II flow cytometer and BD FACSDiva (BD Biosciences).

In vitro differentiation assays. For adipogenic differentiation, cells were cultured in DMEM/10\% FBS supplemented with $1 \mu \mathrm{M}$ dexamethasone, $1 \mu \mathrm{M}$ insulin, $0.5 \mathrm{mM}$ IBMX (all from MilliporeSigma), and 1\% pen/strep for 5 days. Adipogenic differentiation was assessed with Oil Red O staining (ScienCell Research Laboratories) for the presence of lipids according to manufacturer instructions. For osteogenic differentiation, cells were cultured in $10 \mathrm{mM} \beta$-glycerophosphate (Cayman Chemical) and $0.1 \mu \mathrm{M}$ dexamethasone (MilliporeSigma) in Glasgow MEM BHK-21 medium (Gibco, Thermo Fisher Scientific) containing 10\% FBS for up to 4 weeks. Cell cultures were assayed for calcium content by alizarin red staining. For myogenic differentiation cells were seeded on a Matrigel-coated dish at a concentration of $10^{5} \mathrm{cells} / \mathrm{cm}^{2}$ and cultured in DMEM supplemented with $2 \%$ horse serum. For myotube quantification cultures were fixed with 4\% PFA for 10 minutes at room temperature, washed in PBS, and labeled with nuclear DAPI and rabbit anti-human striated MyHC. A MyHC cell that contained more than 3 nuclei with typical morphology was considered a myotube. For induction of fibrosis, LR-PCs (passages 6-7) and $\mathrm{CD} 146^{+} \mathrm{CD} 56^{+}$and $\mathrm{CD} 146^{+} \mathrm{CD} 56^{-}$cells (passages 1-2) were seeded at a concentration of $3 \times 10^{5}$ cells $/ \mathrm{cm}^{2}$ and cultured in DMEM/10\% FBS in the presence or absence of $5 \mathrm{ng} / \mathrm{mL}$ human TGF- $\beta 1$ (R\&D Systems). Triplicates were fixed and stained for collagen production using picrosirius red (Abcam) at 4 days, 2 weeks, and 6 weeks after induction. Extraction of picrosirius red was performed by using $0.1 \mathrm{M} \mathrm{NaOH}$ and collagen quantified using color spectrophotometry ( $\lambda=540 \mathrm{~mm})$.

$R C$ injury model. Massive RC tear surgery was performed as previously described (13). Briefly, the mice (8-10 weeks old) were anesthetized with $2 \%$ isoflurane and oxygen and administered buprenorphine for analgesia, and their right shoulders were sterilely prepared and draped. A 1-cm skin incision was made 
longitudinally over the right glenohumeral joint. The deltoid fibers directly posterior to the deltoid tuberosity were split longitudinally, and the supraspinatus and infraspinatus tendons were identified proximally. A 5-mm section of the tendons was isolated and sharply dissected for the tenotomy procedure (TT). Next, the suprascapular nerve was identified through a $5-\mathrm{mm}$ incision through the trapezius musculature anterior to the lateral scapula, and then the nerve was cut for the denervation procedure (DN). The deltoid muscle and skin were then closed with 5-0 vicryl. For the sham procedure, the same skin incision and muscle dissections were performed, but the tendon transection and denervation procedures were not performed. Mice were sacrificed at 6 or 10 weeks after TTDN for subsequent analyses.

Transplantation of hES LR-PCs and human muscle FAPs. Luciferase-transfected, CM-DiI-labeled LR-PCs (passages 6-8) or human muscle PDGFR- $\alpha^{+} C D 146^{+} C D 56^{-}$FAPs (passages 1-2) were nano-injected $\left(5 \times 10^{5}\right.$ cells $/ 20 \mu \mathrm{L}$ PBS $)$ into the RC muscle of sham- or TTDN-operated mice at 4 days, 2 weeks, and 6 weeks after surgery.

Histology and immunohistochemistry. Infraspinatus and supraspinatus muscles were fixed in $4 \%$ formalin, embedded in paraffin, sectioned, dehydrated, and stained with H\&E for general tissue structure analysis or picrosirius for collagen expression according to manufacturer instructions (Abcam). Images were acquired with an Axio Imager 2 light microscope (Zeiss). For fluorescence microscopy staining, sections were labeled with rabbit anti-human and -mouse $\alpha$-SMA (ab32575, Abcam) overnight at $4^{\circ} \mathrm{C}$ and with secondary antibodies for 30 minutes at room temperature. DAPI (Molecular Probes, 1:1000) was used for nuclei labeling. CM-DiI (Molecular Probes) labeling was performed according to the manufacturer's instructions. Images were acquired with the Axio Imager 2 light microscope (Zeiss).

Quantification of atrophy, fibrosis, and fat content. Regions of interest of histological and immunostained RC sections were imaged, and muscle fiber cross-sectional width was measured using ImageJ (National Institutes of Health). All muscle fibers within each image were measured and averaged for each experimental group. H\&E or DAPI staining was used to visualize, count, and measure the width of myofibers containing central or peripheral nuclei. Fibrosis was quantified by picrosirius red staining and red pixel intensity measurement by Adobe Photoshop, and fraction of fibrosis was calculated by dividing the number of red pixels by the entire number of pixels per area. Adipocytes were counted in H\&E histological RC sections for quantification of fat content.

In vitro atrophy model. $\mathrm{C} 2 \mathrm{C} 12$ culture myogenic induction was carried out in differentiation medium containing DMEM with $2 \%$ horse serum. Control cultures of differentiated myotubes were maintained in DMEM/10\% FBS. LR-PCs were inactivated with $10 \mu \mathrm{g} / \mathrm{mL}$ of mitomycin C for 3 hours and were maintained in DMEM/10\% FBS. Conditioned medium was collected at 24 and 48 hours after medium exchange and centrifuged at $1000 \mathrm{~g}$ to pellet detached cells and large debris. As an in vitro model of atrophy, cultures of myotubes were treated with 10 $\mu \mathrm{M}$ or $100 \mu \mathrm{M}$ DXM for 4 days in the presence or absence of LR-PC-conditioned medium. Starvation-induced atrophy of myotubes was carried out for 4 days without culture refeeding. Control and conditioned mediumtreated cultures were fed every 2 days. For morphological analysis, cultures were fixed, and average diameters of myotubes were measured for each condition at 3 points along the length of the myotube.

Statistics. All data are presented as mean \pm SEM. Single-factor ANOVA was used to compare mean values among study groups (Microsoft Excel 2016). For all analyses, a $P$ value of less than 0.05 was considered statistically significant.

Study approval. The studies were approved by the UCLA Animal Research Committee in the Office of Animal Research Oversight (no. 2012-042 and no. 2014-125), the IRB of UCLA (no. 18-001573), and the Embryonic Stem Cell Research Oversight Committee of UCLA (no. 2006-009). Human deltoid and subscapularis were obtained with written informed consent.

\section{Author contributions}

GMM performed mouse surgeries; designed and executed experiments; collected, analyzed, and interpreted data; and wrote sections of the manuscript. RH, PS, AS, KR, TA, VJH, DJM, GW, JDG, and HX executed experiments; collected, analyzed, and interpreted data; and wrote sections of the manuscript. AP, $\mathrm{BP}, \mathrm{FAP}$, and $\mathrm{AD}$ designed experiments and wrote and critically reviewed the manuscript. BP, FAP, and $\mathrm{AD}$ approved the submitted version of the manuscript. All authors reviewed the manuscript.

\section{Acknowledgments}

This work was supported by the H\&H Lee Surgical Research Scholars Program and the UCLA David Geffen School of Medicine, Department of Orthopaedic Surgery. 
Address correspondence to: Ayelet Dar, Orthopaedic Hospital Research Center, 615 Charles E. Young Drive South, Los Angeles, California 90095, USA. Phone: 310.825.3399; Email: darayelet@gmail.com.

1. Yamaguchi K, Tetro AM, Blam O, Evanoff BA, Teefey SA, Middleton WD. Natural history of asymptomatic rotator cuff tears: a longitudinal analysis of asymptomatic tears detected sonographically. J Shoulder Elbow Surg. 2001;10(3):199-203.

2. Gerber C, Fuchs B, Hodler J. The results of repair of massive tears of the rotator cuff. J Bone Joint Surg Am. 2000;82(4):505-515.

3. Gladstone JN, Bishop JY, Lo IK, Flatow EL. Fatty infiltration and atrophy of the rotator cuff do not improve after rotator cuff repair and correlate with poor functional outcome. Am J Sports Med. 2007;35(5):719-728.

4. Gimbel JA, Mehta S, Van Kleunen JP, Williams GR, Soslowsky LJ. The tension required at repair to reappose the supraspinatus tendon to bone rapidly increases after injury. Clin Orthop Relat Res. 2004;null(426):258-265.

5. Gimbel JA, Van Kleunen JP, Lake SP, Williams GR, Soslowsky LJ. The role of repair tension on tendon to bone healing in an animal model of chronic rotator cuff tears. J Biomech. 2007;40(3):561-568.

6. Gimbel JA, Van Kleunen JP, Mehta S, Perry SM, Williams GR, Soslowsky LJ. Supraspinatus tendon organizational and mechanical properties in a chronic rotator cuff tear animal model. J Biomech. 2004;37(5):739-749.

7. Meyer DC, Gerber C, Von Rechenberg B, Wirth SH, Farshad M. Amplitude and strength of muscle contraction are reduced in experimental tears of the rotator cuff. Am J Sports Med. 2011;39(7):1456-1461.

8. Eliasberg CD, et al. Perivascular stem cells diminish muscle atrophy following massive rotator cuff tears in a small animal model. J Bone Joint Surg Am. 2017;99(4):331-341.

9. Dellavalle A, et al. Pericytes of human skeletal muscle are myogenic precursors distinct from satellite cells. Nat Cell Biol. 2007;9(3):255-267.

10. Uezumi A, Fukada S, Yamamoto N, Takeda S, Tsuchida K. Mesenchymal progenitors distinct from satellite cells contribute to ectopic fat cell formation in skeletal muscle. Nat Cell Biol. 2010;12(2):143-152.

11. Henderson NC, et al. Targeting of $\alpha$ v integrin identifies a core molecular pathway that regulates fibrosis in several organs. Nat Med. 2013;19(12):1617-1624.

12. Murray IR, et al. $\alpha$ v integrins on mesenchymal cells regulate skeletal and cardiac muscle fibrosis. Nat Commun. 2017;8(1):1118.

13. Jensen AR, et al. Neer Award 2018: Platelet-derived growth factor receptor $\alpha$ co-expression typifies a subset of platelet-derived growth factor receptor $\beta$-positive progenitor cells that contribute to fatty degeneration and fibrosis of the murine rotator cuff. J Shoulder Elbow Surg. 2018;27(7):1149-1161.

14. Sacchetti B, et al. No identical "mesenchymal stem cells" at different times and sites: human committed progenitors of distinct origin and differentiation potential are incorporated as adventitial cells in microvessels. Stem Cell Reports. 2016;6(6):897-913.

15. Crisan M, et al. A perivascular origin for mesenchymal stem cells in multiple human organs. Cell Stem Cell. 2008;3(3):301-313.

16. Luzzani CD, Miriuka SG. Pluripotent stem cells as a robust source of mesenchymal stem cells. Stem Cell Rev Rep. 2017;13(1):68-78.

17. Dar A, et al. Multipotent vasculogenic pericytes from human pluripotent stem cells promote recovery of murine ischemic limb. Circulation. 2012;125(1):87-99.

18. Orlova VV, et al. Functionality of endothelial cells and pericytes from human pluripotent stem cells demonstrated in cultured vascular plexus and zebrafish xenografts. Arterioscler Thromb Vasc Biol. 2014;34(1):177-186.

19. Dar A, Itskovitz-Eldor J. Therapeutic potential of perivascular cells from human pluripotent stem cells. J Tissue Eng Regen Med. 2015;9(9):977-987.

20. Kumar A, et al. Specification and diversification of pericytes and smooth muscle cells from mesenchymoangioblasts. Cell Rep. 2017;19(9):1902-1916.

21. Chin CJ, et al. Transcriptionally and functionally distinct mesenchymal subpopulations are generated from human pluripotent stem cells. Stem Cell Reports. 2018;10(2):436-446.

22. Liu X, Manzano G, Kim HT, Feeley BT. A rat model of massive rotator cuff tears. J Orthop Res. 2011;29(4):588-595.

23. Caplan AI. Adult mesenchymal stem cells for tissue engineering versus regenerative medicine. J Cell Physiol. 2007;213(2):341-347.

24. Rohban R, Pieber TR. Mesenchymal stem and progenitor cells in regeneration: tissue specificity and regenerative potential. Stem Cells Int. 2017;2017:5173732.

25. Gumucio JP, et al. Stromal vascular stem cell treatment decreases muscle fibrosis following chronic rotator cuff tear. Int Orthop. 2016;40(4):759-764.

26. Heredia JE, et al. Type 2 innate signals stimulate fibro/adipogenic progenitors to facilitate muscle regeneration. Cell. 2013;153(2):376-388.

27. Uezumi A, et al. Identification and characterization of PDGFR $\alpha+$ mesenchymal progenitors in human skeletal muscle. Cell Death Dis. 2014;5:e1186.

28. Jensen AR, et al. Neer Award 2018: Platelet-derived growth factor receptor $\alpha$ co-expression typifies a subset of platelet-derived growth factor receptor $\beta$-positive progenitor cells that contribute to fatty degeneration and fibrosis of the murine rotator cuff. J Shoulder Elbow Surg. 2018;27(7):1149-1161.

29. Silver MM, Gilbert JJ, Stewart S, Brabyn D, Jung J. Morphologic and morphometric analysis of muscle in X-linked myotubular myopathy. Hum Pathol. 1986;17(11):1167-1178.

30. Anderson JE. Dystrophic changes in mdx muscle regenerating from denervation and devascularization. Muscle Nerve. 1991;14(3):268-279.

31. Lawlor MW, Dechene ET, Roumm E, Geggel AS, Moghadaszadeh B, Beggs AH. Mutations of tropomyosin 3 (TPM3) are common and associated with type 1 myofiber hypotrophy in congenital fiber type disproportion. Hum Mutat. 2010;31(2):176-183.

32. Stitt TN, et al. The IGF-1/PI3K/Akt pathway prevents expression of muscle atrophy-induced ubiquitin ligases by inhibiting FOXO transcription factors. Mol Cell. 2004;14(3):395-403.

33. Park CM, Kim MJ, Kim SM, Park JH, Kim ZH, Choi YS. Umbilical cord mesenchymal stem cell-conditioned media prevent mus- 
cle atrophy by suppressing muscle atrophy-related proteins and ROS generation. In Vitro Cell Dev Biol Anim. 2016;52(1):68-76.

34. Mann CJ, et al. Aberrant repair and fibrosis development in skeletal muscle. Skelet Muscle. 2011;1(1):21.

35. Mueller AA, van Velthoven CT, Fukumoto KD, Cheung TH, Rando TA. Intronic polyadenylation of PDGFR $\alpha$ in resident stem cells attenuates muscle fibrosis. Nature. 2016;540(7632):276-279.

36. Olson LE, Soriano P. Increased PDGFRalpha activation disrupts connective tissue development and drives systemic fibrosis. Dev Cell. 2009;16(2):303-313.

37. Murray IR, et al. Skeletal and cardiac muscle pericytes: Functions and therapeutic potential. Pharmacol Ther. 2017;171:65-74.

38. Gallini R, Lindblom P, Bondjers C, Betsholtz C, Andrae J. PDGF-A and PDGF-B induces cardiac fibrosis in transgenic mice. Exp Cell Res. 2016;349(2):282-290.

39. Kang JR, Gupta R. Mechanisms of fatty degeneration in massive rotator cuff tears. J Shoulder Elbow Surg. 2012;21(2):175-180.

40. Stenmark KR, Davie N, Frid M, Gerasimovskaya E, Das M. Role of the adventitia in pulmonary vascular remodeling. Physiology (Bethesda). 2006;21:134-145.

41. Campagnolo P, et al. Human adult vena saphena contains perivascular progenitor cells endowed with clonogenic and proangiogenic potential. Circulation. 2010;121(15):1735-1745.

42. Cao Z, et al. Targeting the vascular and perivascular niches as a regenerative therapy for lung and liver fibrosis. Sci Transl Med. 2017;9(405):eaai8710

43. Kostallari E, et al. Pericytes in the myovascular niche promote post-natal myofiber growth and satellite cell quiescence. Development. 2015;142(7):1242-1253.

44. Pierantozzi E, et al. Tissue-specific cultured human pericytes: perivascular cells from smooth muscle tissue have restricted mesodermal differentiation ability. Stem Cells Dev. 2016;25(9):674-686.

45. Fry CS, Kirby TJ, Kosmac K, McCarthy JJ, Peterson CA. Myogenic progenitor cells control extracellular matrix production by fibroblasts during skeletal muscle hypertrophy. Cell Stem Cell. 2017;20(1):56-69.

46. Hicks MR, et al. ERBB3 and NGFR mark a distinct skeletal muscle progenitor cell in human development and hPSCs. Nat Cell Biol. 2018;20(1):46-57.

47. Malecova B, et al. Dynamics of cellular states of fibro-adipogenic progenitors during myogenesis and muscular dystrophy. Nat Commun. 2018;9(1):3670.

48. Hsu CY, et al. Comparison of human tissue microarray to human pericyte transcriptome yields novel perivascular cell markers. Stem Cells Dev. 2019;28(18):1214-1223.

49. Ilic D, Devito L, Miere C, Codognotto S. Human embryonic and induced pluripotent stem cells in clinical trials. Br Med Bull. 2015;116:19-27.

50. Xi H, et al. In vivo human somitogenesis guides somite development from hPSCs. Cell Rep. 2017;18(6):1573-1585 